\title{
Fluctuations of the critical Casimir force
}

\author{
Markus Gross, ${ }^{1,2,{ }^{*}}$ Andrea Gambassi, ${ }^{3}$ and S. Dietrich ${ }^{1,2}$ \\ ${ }^{1}$ Max-Planck-Institut für Intelligente Systeme, Heisenbergstraße 3, 70569 Stuttgart, Germany \\ ${ }^{2}$ IV. Institut für Theoretische Physik, Universität Stuttgart, Pfaffenwaldring 57, 70569 Stuttgart, Germany \\ ${ }^{3}$ SISSA-International School for Advanced Studies and INFN, via Bonomea 265, 34136 Trieste, Italy
}

(Received 24 November 2020; accepted 20 May 2021; published 14 June 2021)

\begin{abstract}
The critical Casimir force (CCF) arises from confining fluctuations in a critical fluid and thus it is a fluctuating quantity itself. While the mean CCF is universal, its (static) variance has previously been found to depend on the microscopic details of the system which effectively set a large-momentum cutoff in the underlying field theory, rendering it potentially large. This raises the question how the properties of the force variance are reflected in experimentally observable quantities, such as the thickness of a wetting film or the position of a suspended colloidal particle. Here, based on a rigorous definition of the instantaneous force, we analyze static and dynamic correlations of the $\mathrm{CCF}$ for a conserved fluid in film geometry for various boundary conditions within the Gaussian approximation. We find that the dynamic correlation function of the CCF is independent of the momentum cutoff and decays algebraically in time. Within the Gaussian approximation, the associated exponent depends only on the dynamic universality class but not on the boundary conditions. We furthermore consider a fluid film, the thickness of which can fluctuate under the influence of the time-dependent CCF. The latter gives rise to an effective non-Markovian noise in the equation of motion of the film boundary and induces a distinct contribution to the position variance. Within the approximations used here, at short times, this contribution grows algebraically in time whereas, at long times, it saturates and contributes to the steady-state variance of the film thickness.
\end{abstract}

DOI: 10.1103/PhysRevE.103.062118

\section{INTRODUCTION}

A fluid close to a continuous phase transition exhibits remarkable universal properties, such as long-ranged fluctuations and scale invariance [1]. A core element of criticality is the notion of an order parameter (OP) $\phi$, which, e.g., in the case of a binary liquid mixture is proportional to the deviation of the concentration $c_{A}$ of species $A$ from its critical value $c_{A, c}$, i.e., $\phi \propto c_{A}-c_{A, c}$. Confining a critical fluid leads to a critical Casimir force (CCF) acting on the confining boundaries [2-4]. Such a situation arises, e.g., when two colloidal particles immersed in a critical solvent come close to another or if one colloidal particle approaches a container wall of the sample. CCFs thus provide means to control the aggregation behavior of colloidal suspensions [5] and are, accordingly, not only of theoretical but also of highly practical interest. Consequently, CCFs and, more generally, Casimir-type fluctuation-induced forces have been extensively studied in as well as out of equilibrium (see, e.g., Refs. [6-11] and references therein).

\footnotetext{
*gross@is.mpg.de
}

Published by the American Physical Society under the terms of the Creative Commons Attribution 4.0 International license. Further distribution of this work must maintain attribution to the author(s) and the published article's title, journal citation, and DOI. Open access publication funded by the Max Planck Society.
Previous studies mainly focused on the mean value of the thermally averaged $\mathrm{CCF}$, which is finite and universal, i.e., it does not depend on the specific material considered, but only on the bulk and surface universality class [12]. ${ }^{1}$ However, since the CCF arises from fluctuations of the OP, it is itself a fluctuating quantity. In Ref. [16] the equilibrium fluctuations of the CCF in a generic Gaussian medium subject to Dirichlet boundary conditions (BCs) have been investigated theoretically, and it has been shown that the static equilibrium variance depends in a nonuniversal way on the microscopic details of the model (specifically, on the microscopic length scale below which the continuum description breaks down). These findings were confirmed analytically and via Monte Carlo simulations of lattice models for the case of periodic BCs [17]. The variance of the CCF acting on membrane inclusions has been found to be strongly cutoff dependent as well [18]. In order to understand these results, we note that the (instantaneous) CCF $\mathcal{K}$ (per area) is given by the difference between the pressures acting on the two sides of a boundary, $\mathcal{K}=\mathcal{P}_{f}-\mathcal{P}_{b}$, where the subscripts refer to film and $b$ ulk (a notion to be specified below; see also Fig. 1). Concerning the mean value $\langle\mathcal{K}\rangle$, all cutoff-dependent quantities present in $\mathcal{P}_{f, b}$ cancel in the difference, giving rise to a

\footnotetext{
${ }^{1}$ This notion of universality applies to a fluid formed by particles with short-ranged interactions. In the presence of van der Waals forces, in particular, the CCF can exhibit a nonuniversal behavior [13-15].
} 


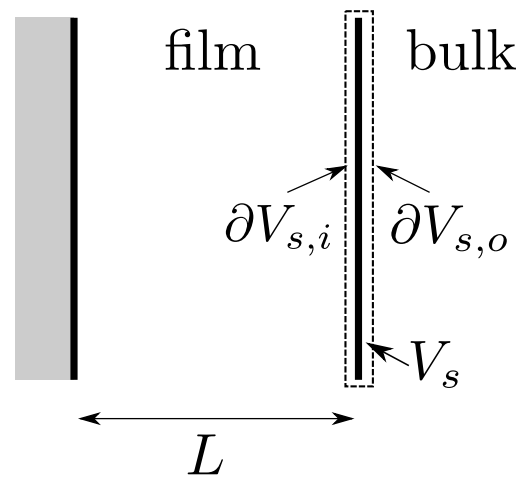

FIG. 1. Sketch of the situation considered in this study. A film of thickness $L$ is enclosed by two boundaries (thick black lines) each of surface area $A$ (spanning the plane perpendicular to the figure). The right boundary is enclosed by a thin imaginary volume $V_{s}$. The CCF acting on the right boundary is obtained from an integration of the stress tensor over the surface of $V_{s}$, which consists of an inner $\left(\partial V_{s, i}\right)$ and an outer part $\left(\partial V_{s, o}\right)$ [see Eq. (2.18)]. The film and the bulk consist of the same liquid, whereas the gray area corresponds to a solid.

finite and in fact universal quantity. In contrast, the variance $\left\langle(\Delta \mathcal{K})^{2}\right\rangle=\left\langle\Delta \mathcal{P}_{f}^{2}\right\rangle+\left\langle\Delta \mathcal{P}_{b}^{2}\right\rangle$ (where $\Delta \mathcal{K} \equiv \mathcal{K}-\langle\mathcal{K}\rangle$ ) is the sum of the individual variances of $\mathcal{P}_{f}$ and $\mathcal{P}_{b}$ (assuming statistical independence of film and bulk) and thus no cancellation of divergences can occur [16].

Notably, the detailed form of the cutoff dependence of the variance implies a divergence in the strict continuum limit. The corresponding huge variances predicted in this way for the CCF seemingly stand in contrast to the fact that experiments on CCFs in wetting films [19-22] or in colloidal systems $[5,23]$ did not observe such giant fluctuations. Colloidal particles in critical bulk fluids rather show essentially standard Brownian diffusion with an effective diffusivity modified due to the fluctuations of the field [24-26]. Moreover, near a wall, colloids diffusively explore the corresponding effective potential induced by the mean CCF $[27,28]$.

Divergent equilibrium variances of the force have also been obtained for the quantum-electrodynamical Casimir effect [29]. However, it has been shown that the divergence problem is less severe or even absent when considering temporally averaged quantities [16,29]. In fact, such a temporal average is indispensable due to the necessarily finite temporal resolution of a measurement apparatus. This issue can also be appreciated in view of a Langevin description of a Brownian particle [30]: while the random force has correlations proportional to a Dirac $\delta$ function and thus is strongly divergent, the resulting observable quantities, such as the position distribution of the particle, are finite [24-26]. We finally remark that the static variance of thermal van der Waals forces between dielectric slabs has been found to be finite and independent of a microscopic cutoff, without the necessity for temporal averaging [31].

In this study, based on the nonequilibrium stress tensor formalism [9,32-34], we investigate static and dynamic equilibrium fluctuations of the CCF in film geometry for various non-symmetry-breaking BCs. We focus on the Gaussian ap- proximation. Simulations of lattice models [17] have shown that it constitutes an accurate description of the (static) stress probability distribution. In accordance with Refs. $[16,17]$ we confirm the generic nonuniversal and divergent character of the static variance of the CCF acting on a fixed boundary. Going beyond previous studies, we show that the dynamic correlations of the CCF are finite and decay algebraically in time with exponents depending only on the spatial dimensionality and the dynamic universality class. We furthermore consider a movable film boundary subject to the fluctuations of the CCF. We find that the mean-squared displacement of the boundary is, at short times, characterized by an algebraic growth (with possible logarithmic corrections), while, at long times, it saturates at a finite value due to the confining potential stemming from the CCF. Accordingly, the strong divergences of the static variance of the CCF as function of the cutoff do not show up in its dynamic correlations or in experimentally observable quantities such as the position of the film boundary.

We present our study as follows: In Sec. II, we introduce the model and briefly review the formalism required to determine time-dependent CCFs. In Sec. III, we analyze static and dynamic fluctuations of the CCF in the case of a film with fixed boundaries. This constraint is released in Sec. IV, where we consider the thermal fluctuations of the film thickness. A summary of the findings of this study is provided in Sec. V.

\section{PRELIMINARIES}

\section{A. Order-parameter dynamics}

In thermal equilibrium at temperature $T$ [defined here in units of $k_{B}$, i.e., $\left.T=T_{\text {phys }} /\left(1 / k_{B}\right)\right]$ and in $d$ spatial dimensions, the OP $\phi$ follows the probability distribution

$$
P_{\mathrm{eq}}[\phi] \sim e^{-\mathcal{F}[\phi] / T},
$$

with the Hamiltonian $\left[\mathbf{r}=\left(r_{1}, \ldots, r_{d}\right)\right]$

$$
\begin{aligned}
\mathcal{F}[\phi] \equiv & \int_{V} d^{d} r \mathcal{H}(\mathbf{r}, L, \phi(\mathbf{r}), \nabla \phi(\mathbf{r}), \tau), \\
\mathcal{H}(\mathbf{r}, L, \phi(\mathbf{r}), \nabla \phi(\mathbf{r}), \tau)= & \mathcal{H}_{b}(\phi(\mathbf{r}), \nabla \phi(\mathbf{r}), \tau) \\
& +\mathcal{H}_{s}(\mathbf{r}, L, \phi(\mathbf{r}), \nabla \phi(\mathbf{r})) ;
\end{aligned}
$$

accordingly, $\mathcal{F}$ and $T$ have the dimension energy and $\mathcal{F} / T$ is dimensionless. For the bulk Hamiltonian density $\mathcal{H}_{b}$ we consider a Gaussian Landau-Ginzburg form

$$
\mathcal{H}_{b}(\phi, \nabla \phi, \tau)=\frac{1}{2}(\nabla \phi)^{2}+\frac{\tau}{2} \phi^{2},
$$

with $(\nabla \phi)^{2}=\sum_{\alpha=1}^{d}\left(\partial_{\alpha} \phi\right)^{2}, \partial_{\alpha}=\frac{\partial}{\partial r_{\alpha}} \equiv \nabla_{\alpha}$, and the temperature parameter

$$
\tau=\left(\xi_{+}^{(0)}\right)^{-2} \stackrel{\tau}{ }
$$

which is directly proportional to the reduced temperature

$$
\stackrel{\imath}{\tau} \equiv \frac{T-T_{c}}{T_{c}}>0,
$$

where $T_{c}$ denotes the (bulk) critical temperature. Furthermore, $\xi_{+}^{(0)}$ denotes a nonuniversal critical amplitude defined via the correlation length $\xi$ of the fluctuations as

$$
\xi\left(\stackrel{\circ}{\tau} \rightarrow 0^{+}\right)=\xi_{+}^{(0)} \stackrel{\tau}{-v}^{-v}=\left(\xi_{+}^{(0)}\right)^{1-2 v} \tau^{-v},
$$


with a bulk critical exponent $v$. Within the Gaussian approximation, one has $v=\frac{1}{2}$, such that Eq. (2.6) reduces to $\xi=$ $1 / \sqrt{\tau}$. For typical simple fluids one finds $\xi_{+}^{(0)} \approx 1 \mathrm{~nm}$ [21]. For the surface Hamiltonian density $\mathcal{H}_{s}$ we do not assume a specific form, except that it is strongly localized at a boundary; e.g., for a planar surface at $z=L$, it takes the form

$$
\mathcal{H}_{s}(\mathbf{r}, L, \phi, \nabla \phi)=\delta(z-L) U_{s}(\phi, \nabla \phi)
$$

with a potential $U_{s}$. We consider the thin film geometry with macroscopically large lateral directions and, accordingly, we decompose the $d$-dimensional vector $\mathbf{r}$ as $\mathbf{r}=\left\{\mathbf{r}_{\|}=\right.$ $\left.\left(r_{1}, \ldots, r_{d-1}\right), z\right\}$. The following BCs of the OP are considered in the transverse direction $z$ :

$$
\begin{array}{cl}
\text { periodic : } & \phi\left(\mathbf{r}_{\|}, z\right)=\phi\left(\mathbf{r}_{\|}, z+L\right), \\
\text { Dirichlet : } & \phi\left(\mathbf{r}_{\|}, 0\right)=0=\phi\left(\mathbf{r}_{\|}, L\right),
\end{array}
$$

and

$$
\text { Neumann : }\left.\quad \partial_{z} \phi\left(\mathbf{r}_{\|}, z\right)\right|_{z=0}=0=\left.\partial_{z} \phi\left(\mathbf{r}_{\|}, z\right)\right|_{z=L} .
$$

Denoting the physical time by $\tilde{t}$ [to be distinguished from the rescaled one, see Eq. (2.11) below], the OP dynamics is taken to be described by model B [35]:

$$
\partial_{\bar{t}} \phi=\gamma \nabla^{2} \mu(\phi)+\bar{\zeta},
$$

where $\gamma$ is a mobility coefficient and

$$
\mu(\phi) \equiv \frac{\delta \mathcal{F}(\tau ;[\phi])}{\delta \phi}=\frac{\partial \mathcal{H}}{\partial \phi}-\sum_{j=1}^{d} \nabla_{j}\left(\frac{\partial \mathcal{H}}{\partial \nabla_{j} \phi}\right)
$$

is the chemical potential. Far away from a boundary, one has $\mu=-\nabla^{2} \phi+\tau \phi$. Furthermore, $\bar{\zeta}$ is a Gaussian white noise with zero mean and correlations $\left\langle\bar{\zeta}(\mathbf{r}, \tilde{t}) \bar{\zeta}\left(\mathbf{r}^{\prime}, \tilde{t}^{\prime}\right)\right\rangle=$ $-2 T \gamma \nabla^{2} \delta\left(\mathbf{r}-\mathbf{r}^{\prime}\right) \delta\left(\tilde{t}-\tilde{t}^{\prime}\right)$. The form of Eq. (2.9) and of the noise ensure local conservation of the OP in an unconfined system. In order to guarantee local and global OP conservation in the presence of boundaries, the flux $\boldsymbol{J}=-\gamma \nabla \mu+\mathcal{N}$ of the OP (with noise $\mathcal{N}$ such that $\nabla \cdot \mathcal{N}=\bar{\zeta}$ ) must vanish at the confining surfaces of the system, i.e., $J_{z}=0$ for $z=0, L{ }^{2}$ This is ensured by periodic or Neumann BCs, which we shall henceforth use in the dynamic model. ${ }^{3}$ In order to simplify the notation, we follow Ref. [9] and remove the temperature $T$ from the description by introducing a rescaled OP field $\phi / T^{1 / 2}$ and, additionally, introduce a rescaled time

$$
t=\gamma \tilde{t}
$$

having the dimension $[t] \sim[L]^{4}$. Accordingly, Eq. (2.9) turns into

$$
\partial_{t} \phi=-\nabla^{4} \phi+\tau \nabla^{2} \phi+\zeta,
$$

with a Gaussian noise $\zeta \equiv \bar{\zeta} / T^{1 / 2} \gamma$ correlated as

$$
\left\langle\zeta(\mathbf{r}, t) \zeta\left(\mathbf{r}^{\prime}, t^{\prime}\right)\right\rangle=-2 \nabla^{2} \delta\left(\mathbf{r}-\mathbf{r}^{\prime}\right) \delta\left(t-t^{\prime}\right) .
$$

\footnotetext{
${ }^{2}$ In fact, we require the deterministic and stochastic fluxes $\mathbf{J}_{d}=$ $-\gamma \nabla \mu$ and $\mathbf{J}_{s}=\mathcal{N}$ to both vanish independently at the boundaries.

${ }^{3}$ Standard Dirichlet BCs do not constrain the derivatives of $\phi$ at the boundary and therefore entail in general a nonvanishing flux $\mathbf{J}$ across a boundary. Thus, they are not considered here; see Refs. $[9,36]$ for further discussion.
}

\section{B. Forces}

The instantaneous CCF alluded to in the Introduction is defined in terms of a dynamic stress tensor which can be derived for generic Landau-Ginzburg type of models [32]. The associated formalism has been developed further in Ref. [33] and has been applied in Refs. [9,34] for the case of a quench of a conserved fluid at criticality. Notably, the expressions for the stress tensor in and out of equilibrium are, in general, different. ${ }^{4}$ The concept of a dynamic stress tensor allows one to rigorously define an CCF at any instant during the time evolution of the system.

The determination of time-dependent forces on boundaries within model B is detailed in Refs. [9,34]; in the following we provide only the essential equations. The dynamical stress tensor is given by [34]

$$
\overline{\mathcal{T}}_{\alpha \beta} \equiv \mathcal{T}_{\alpha \beta}+\mu \phi \delta_{\alpha \beta},
$$

which is expressed here in terms of the standard (grand canonical) stress tensor

$$
\mathcal{T}_{\alpha \beta}=\frac{\partial \mathcal{H}}{\partial \nabla_{\alpha} \phi} \nabla_{\beta} \phi-\delta_{\alpha \beta} \mathcal{H}
$$

The last term in Eq. (2.14) involving the chemical potential $\mu$ [Eq. (2.10)] generically arises in a nonequilibrium situation for a system with no-flux BCs. Considering the right boundary of the film at position $z=L$ as shown in Fig. 1, the instantaneous generalized force $\mathcal{K}_{\alpha}$ (per area $A$ ) in direction $\alpha$ of the surface is defined as

$$
\mathcal{K}_{\alpha}=-\frac{1}{A} \frac{\partial \mathcal{F}}{\partial L},
$$

which can be transformed into [34]

$$
\mathcal{K}_{\alpha} A=-\int_{\partial V_{s}} d^{d-1} s_{\beta} \overline{\mathcal{T}}_{\alpha \beta}+\int_{V_{s}} d^{d} r\left(\nabla_{\alpha} \mu\right) \phi,
$$

where $V_{s}$ is an infinitesimally thin volume enclosing the surface. If $\phi$ fulfills no-flux BCs or Dirichlet BCs, the last term in Eq. (2.17) vanishes. We note that, due to the localized nature of $\mathcal{H}_{s}$ [Eq. (2.7)], effectively only the bulk Hamiltonian density $\mathcal{H}_{b}$ enters into $\overline{\mathcal{T}}_{\alpha \beta}$ in Eq. (2.17). In the case of periodic BCs, Eq. (2.17) has to be suitably symmetrized (see Ref. [9]). Since the resulting expression for the CCF is identical to omitting the last term in Eq. (2.17), we do not separately deal with this aspect. In general, the instantaneous CCF (per area) $\mathcal{K} \equiv \mathcal{K}_{z}$ acting on a boundary at position $z$ of the film is obtained as

$$
\mathcal{K}(z, t)=\mathcal{P}_{f}(z, t)-\mathcal{P}_{b}(z, t),
$$

where

$$
\mathcal{P}_{f, b}(z, t)=\frac{1}{A} \int_{\partial V_{s, i / o}} d s_{z}\left(\mathbf{r}_{\|}, z\right) \overline{\mathcal{T}}_{z z}\left(\mathbf{r}_{\|}, z, t\right)
$$

represents the film and the bulk pressure; $\partial V_{s, i / o}$, correspondingly, denotes the bounding surface of the inner and outer fluid and $\mathbf{r}_{\|}$is the location of the $(d-1)$-dimensional surface

\footnotetext{
${ }^{4} \mathrm{An}$ exception is the case of Dirichlet BCs, for which the timedependent CCF in film geometry has been studied in Refs. [37,38].
} 
element $d s_{z}$. We emphasize that the quantity $\mathcal{K}$ is actually a pressure. However, to stay in line with the convention employed in most of the literature, we use the term Casimir force throughout this study. In equilibrium, we assume the boundaries of the film to be fixed at $z \in\{0, L\}$. However, in order to facilitate later extensions of the model, in our formalism we shall keep $z$ general. For the Landau-Ginzburg Hamiltonian density of Eq. (2.3) and upon using Eq. (2.10), the dynamical stress tensor takes the form

$$
\overline{\mathcal{T}}_{z z}=\frac{1}{2}\left(\partial_{z} \phi\right)^{2}-\frac{1}{2} \sum_{\alpha=1}^{d-1}\left(\partial_{\alpha} \phi\right)^{2}-\phi \nabla^{2} \phi+\frac{1}{2} \tau \phi^{2},
$$

which, as noted after Eq. (2.17), depends only on bulk terms. The mean CCF (per area) acting on a surface at location $z$ follows from Eq. (2.18) as

$$
\begin{aligned}
\langle\mathcal{K}(z, t)\rangle & =\left\langle\mathcal{P}_{f}(z)-\mathcal{P}_{b}(z)\right\rangle \equiv \frac{1}{A} \int_{\partial V_{s, i}} d s_{z}\left(\mathbf{r}_{\|}, z\right)\left\langle\bar{T}_{z z}\left(\mathbf{r}_{\|}, z, t\right)\right\rangle-\{\text { bulk }\} \\
& =\left[\frac{1}{2}\left\langle\left[\partial_{z} \phi\left(\mathbf{r}_{\|}, z, t\right)\right]^{2}\right\rangle-\frac{1}{2} \sum_{\alpha=1}^{d-1}\left\langle\left[\partial_{\alpha} \phi\left(\mathbf{r}_{\|}, z, t\right)\right]^{2}\right\rangle-\left\langle\phi\left(\mathbf{r}_{\|}, z, t\right) \sum_{\alpha=1}^{d} \partial_{\alpha}^{2} \phi\left(\mathbf{r}_{\|}, z, t\right)\right\rangle\right]_{\substack{\mathbf{r}_{\|}=0 \\
z=0, L}}-\{\text { bulk }\}
\end{aligned}
$$

where " $\{$ bulk\}" denotes the corresponding expression evaluated in the bulk, which is obtained by integrating over $\partial V_{s, o}$ and calculating statistical averages with the corresponding bulk distribution of $\phi$.

\section{Order-parameter correlation functions}

Here, we summarize some useful expressions for and properties of the equilibrium two-point two-time OP correlation function $\mathcal{C}\left(\mathbf{r}, \mathbf{r}^{\prime}, t\right) \equiv\left\langle\phi(\mathbf{r}, t) \phi\left(\mathbf{r}^{\prime}, 0\right)\right\rangle=\mathcal{C}\left(\mathbf{r}_{\|}-\right.$ $\left.\mathbf{r}_{\|}^{\prime}, z, z^{\prime}, t\right)$, where the latter equality follows from translational invariance in the lateral directions. The time-independent, static limit of $\mathcal{C}$ is denoted by $\mathcal{C}_{\text {st }}$ and follows as $\mathcal{C}_{\text {st }}\left(\mathbf{r}_{\|}, z, z^{\prime}\right)=$ $\mathcal{C}\left(\mathbf{r}_{\|}, z, z, t \rightarrow 0\right)$.

\section{Bulk correlation function}

The equilibrium bulk correlation function for model $\mathrm{B}$ is given by (see, e.g., Ref. [9])

$$
\mathcal{C}_{b}(r, t) \equiv \mathcal{C}_{b}(\mathbf{r}, t)=\int \frac{d^{d} q}{(2 \pi)^{d}} e^{i \mathbf{q} \cdot \mathbf{r}} \frac{e^{-\mathbf{q}^{2}\left(\mathbf{q}^{2}+\tau\right) t}}{\mathbf{q}^{2}+\tau}
$$

which, as indicated by the notation, is spatially isotropic. ${ }^{5}$ In the static equilibrium case $(t=0)$ for $d>2$ and at bulk criticality $(\tau=0)$, one has

$$
\mathcal{C}_{\mathrm{b}, \mathrm{st}}(r)=\frac{\Gamma(d / 2-1)}{4 \pi^{d / 2} r^{d-2}} \quad(d>2) .
$$

In $d=2$ dimensions, the static bulk correlation function diverges logarithmically for large $r$ [9].

We now analyze $\mathcal{C}_{b}$ [Eq. (2.22)] for $d>2$ in certain relevant limits. Due to the exponential in the expression for $\mathcal{C}_{b}$, the integrand in Eq. (2.22) gives significant contributions only if $\mathbf{q}^{4} t+\mathbf{q}^{2} \tau t \lesssim 1$. There are two characteristic asymptotic regimes depending on $\mathbf{q}^{2} \ll \tau$ or $\mathbf{q}^{2} \gg \tau$, as can be seen by splitting the integral in Eq. (2.22) accordingly:

$$
\begin{aligned}
\mathcal{C}_{b}(r, t) & \simeq \frac{1}{\tau} \int_{|\mathbf{q}| \ll \sqrt{\tau}} \frac{d^{d} q}{(2 \pi)^{d}} e^{i \mathbf{q} \cdot \mathbf{r}-\mathbf{q}^{2} \tau t}+\int_{|\mathbf{q}| \gg \sqrt{\tau}} \frac{d^{d} q}{(2 \pi)^{d}} \mathbf{q}^{-2} e^{i \mathbf{q} \cdot \mathbf{r}-\mathbf{q}^{4} t} \\
& =t^{-d / 2} \tau^{-d / 2-1} \int_{|\hat{\mathbf{q}}| \ll \tau t^{1 / 2}} \frac{d^{d} \hat{q}}{(2 \pi)^{d}} e^{i \hat{\mathbf{q}} \cdot \mathbf{r} / \sqrt{\tau t}-\hat{\mathbf{q}}^{2}}+t^{1 / 2-d / 4} \int_{|\hat{\mathbf{q}}| \gg \sqrt{\tau t^{1 / 2}}} \frac{d^{d} \hat{q}}{(2 \pi)^{d}} \hat{\mathbf{q}}^{-2} e^{i \hat{\mathbf{q}} \cdot \mathbf{r} / t^{1 / 4}-\hat{\mathbf{q}}^{4}} .
\end{aligned}
$$

If $\tau t^{1 / 2} \gg 1$, i.e., for long times $t \gg \tau^{-2}$, the second term on the right-hand side of Eq. (2.24) is exponentially suppressed, and we can obtain an asymptotic estimate of $\mathcal{C}_{b}$ by extending the integration range in the first term to all $\mathbf{q}$ :

$$
\begin{aligned}
\left.\mathcal{C}_{b}(r, t)\right|_{t \gg \tau^{-2}} & \simeq t^{-d / 2} \tau^{-d / 2-1} \int \frac{d^{d} \hat{q}}{(2 \pi)^{d}} e^{i \mathbf{q} \cdot \mathbf{r} /(\tau t)^{1 / 2}-\hat{\mathbf{q}}^{2}} \\
& =(4 \pi)^{-d / 2} t^{-d / 2} \tau^{-d / 2-1} \exp \left(-\frac{r^{2}}{4 \tau t}\right) .
\end{aligned}
$$

On the other hand, for $\tau t^{1 / 2} \ll 1$, i.e., at short times $t \ll \tau^{-2}$, the second term in Eq. (2.24) dominates, which can be shown as follows: for $\tau t^{1 / 2} \ll 1$, the first term in Eq. (2.24) can be approximated as $t^{-d / 2} \tau^{-d / 2-1} \int \frac{d^{d} \hat{q}}{(2 \pi)^{d}} \exp (i \hat{\mathbf{q}} \cdot \mathbf{r} / \sqrt{\tau t}) \simeq$ $t^{-d / 2} \tau^{-d / 2-1} \sqrt{\tau t} \tilde{\delta}^{(d)}(\mathbf{r})=\tau^{-1}$, where $\tilde{\delta}^{(d)}$ stands for an approximation of the Dirac $\delta$ function. The ratio of the first and the

\footnotetext{
${ }^{5}$ Note that, due to the time-reversal symmetry of the model, all correlation functions in fact depend on $|t|$ instead of $t$.
} 
second term follows as $\left(\tau^{2} t\right)^{d / 4-1 / 2} / \tau^{d / 2}$. Accordingly, for $d>2$, the first term is indeed negligible at short times. The bulk correlation function can be asymptotically estimated by extending the integration in the second term in Eq. (2.24) over the whole range of $\hat{\mathbf{q}}$, resulting in [9]

$$
\begin{aligned}
\left.\mathcal{C}_{b}(r, t)\right|_{\tau=0}= & t^{1 / 2-d / 4} \int \frac{d^{d} \hat{q}}{(2 \pi)^{d}} \hat{\mathbf{q}}^{-2} e^{i \hat{\mathbf{q}} \cdot\left(\mathbf{r} / t^{1 / 4}\right)-\hat{\mathbf{q}}^{4}} \\
= & \frac{2^{d / 4-1 / 2} \pi^{1 / 2-d / 2}}{d \Gamma(1 / 2+d / 4) r^{d-2}} \psi^{d / 4-1 / 2}\left[\frac{d \Gamma(d / 4-1 / 4)}{8 \Gamma(d / 4)}{ }_{1} F_{3}\left(\frac{d}{4}-\frac{1}{2} ; \frac{1}{2}, \frac{1}{2}+\frac{d}{4}, \frac{d}{4} ; \psi\right)\right. \\
& \left.\quad-\sqrt{\frac{\psi}{2}}{ }_{1} F_{3}\left(\frac{d}{4} ; \frac{3}{2}, \frac{1}{2}+\frac{d}{4}, 1+\frac{d}{4} ; \psi\right)\right], \quad \psi \equiv \frac{r^{4}}{256 t},
\end{aligned}
$$

where ${ }_{1} F_{3}$ is a generalized hypergeometric function [39] and, according to Eq. (2.11), the scaling variable $\psi$ is dimensionless.

\section{Film correlation function}

In a thin film with periodic BCs, translational invariance implies $\mathcal{C}^{(\mathrm{p})}\left(\mathbf{r}_{\|}, z, z^{\prime}, t\right)=\mathcal{C}^{(\mathrm{p})}\left(\mathbf{r}_{\|}, z-z^{\prime}, t\right)$ and the two-time correlation function is given by

$$
\begin{aligned}
\mathcal{C}^{(\mathrm{p})}\left(\mathbf{r}_{\|}, z-z^{\prime}, t\right)= & \frac{1}{L} \sum_{n \in \mathbb{Z}} \int \frac{d^{d-1} p}{(2 \pi)^{d-1}} e^{i \mathbf{p} \cdot \mathbf{r}_{\|}+i k_{n}^{(\mathrm{p})}\left(z-z^{\prime}\right)} \\
& \times S\left(|\mathbf{p}|, k_{n}^{(\mathrm{p})}, t\right)
\end{aligned}
$$

where

$$
k_{n}^{(\mathrm{p})} \equiv \frac{2 \pi n}{L}, \quad n=0, \pm 1, \pm 2, \ldots
$$

and

$$
S(p, k, t) \equiv \frac{e^{-\left(p^{2}+k^{2}\right)\left(p^{2}+k^{2}+\tau\right) t}}{p^{2}+k^{2}+\tau} .
$$

For Neumann and Dirichlet BCs, respectively, one has

$$
\begin{aligned}
\mathcal{C}^{(\mathbb{D})}\left(\mathbf{r}_{\|}, z, z^{\prime}, t\right)= & \frac{1}{L} \sum_{n \in\left\{\begin{array}{l}
\mathbb{N}_{0} \\
\mathbb{N}^{\prime}
\end{array}\right\}} \int \frac{d^{d-1} p}{(2 \pi)^{d-1}} e^{i \mathbf{p} \cdot \mathbf{r}_{\|}} \\
& \times\left\{\begin{array}{c}
\cos \left(k_{n}^{(\mathrm{N})} z\right) \cos \left(k_{n}^{(\mathrm{N})} z^{\prime}\right) \\
\sin \left(k_{n}^{(\mathrm{D})} z\right) \sin \left(k_{n}^{(\mathrm{D})} z^{\prime}\right)
\end{array}\right\} S\left(|\mathbf{p}|, k_{n}^{(\mathrm{N} / \mathrm{D})}, t\right),
\end{aligned}
$$

with the eigenmodes

$$
\begin{aligned}
& k_{n}^{(\mathrm{N})} \equiv \frac{\pi n}{L}, \quad n=0,1,2, \ldots, \\
& k_{n}^{(\mathrm{D})} \equiv \frac{\pi n}{L}, \quad n=1,2, \ldots
\end{aligned}
$$

Since (standard) Dirichlet BCs are not flux conserving [see the discussion after Eq. (2.10)], we shall consider these BCs only in the static limit given by setting $t=0$ in Eq. (2.30). By means of the Poisson resummation formula, the above correlation functions can be expressed in terms of $\mathcal{C}_{b}$ as (see, e.g., Refs. [9,37])

$$
\begin{aligned}
\mathcal{C}^{(\mathrm{p})}\left(\mathbf{r}_{\|}, z, t\right) & =\sum_{m=-\infty}^{\infty} \mathcal{C}_{b}\left(\left\{\mathbf{r}_{\|}, z+m L\right\}, t\right), \\
\mathcal{C}^{(\mathrm{N})}\left(\mathbf{r}_{\|}, z, z^{\prime}, t\right) & =\left.\mathcal{C}^{(\mathrm{p})}\left(\mathbf{r}_{\|}, z-z^{\prime}, t\right)\right|_{2 L}+\left.\mathcal{C}^{(\mathrm{p})}\left(\mathbf{r}_{\|}, z+z^{\prime}, t\right)\right|_{2 L}, \\
\mathcal{C}^{(\mathrm{D})}\left(\mathbf{r}_{\|}, z, z^{\prime}, t\right) & =\left.\mathcal{C}^{(\mathrm{p})}\left(\mathbf{r}_{\|}, z-z^{\prime}, t\right)\right|_{2 L}-\left.\mathcal{C}^{(\mathrm{p})}\left(\mathbf{r}_{\|}, z+z^{\prime}, t\right)\right|_{2 L},
\end{aligned}
$$

where, as indicated by the subscript, $\mathcal{C}^{(\mathrm{p})}$ on the right-hand side of Eqs. (2.32b) and (2.32c) are obtained from Eq. (2.32a) by the replacement $L \mapsto 2 L$. The bulk scaling behavior at short times, expressed in Eq. (2.26), lends itself for being generalized to that of a film:

$$
\begin{aligned}
& \mathcal{C}\left(\mathbf{r}_{\|}, z, z^{\prime}, t \ll \tau^{-2}, \tau, L\right) \\
& \quad=t^{-(d-1) / 4} \widehat{\mathcal{C}}\left(\mathbf{r}_{\|} t^{-1 / 4}, z t^{-1 / 4}, z^{\prime} t^{-1 / 4}, L t^{-1 / 4}\right),
\end{aligned}
$$

with a scaling function $\widehat{\mathcal{C}}$, the explicit expression of which is not needed in the following but can in principle be obtained from Eqs. (2.26) and (2.32).

\section{Further properties and relations}

We close this section by collecting a number of relations useful for later developments in this study. Owing to the linearity of the statistical average, one has

$$
\left\langle\partial_{\alpha}^{m} \phi(\mathbf{r}) \partial_{\beta}^{\prime n} \phi\left(\mathbf{r}^{\prime}\right)\right\rangle=\partial_{\alpha}^{m} \partial_{\beta}^{\prime n} \mathcal{C}\left(\mathbf{r}, \mathbf{r}^{\prime}, t\right),
$$

where $\alpha, \beta \in\{1, \ldots, d\}$ and $\partial_{\alpha}^{\prime} \equiv \partial / \partial_{r_{\alpha}^{\prime}}$. Analogously, the Fourier and Poisson representations of the correlation function for the BCs considered here [see Eqs. (2.22), (2.27), (2.30), and (2.32)] render

$$
\left.\partial_{z} \mathcal{C}\left(\mathbf{r}_{\|}, z, z^{\prime}, t\right)\right|_{z=z^{\prime}=0}=0=\left.\partial_{z^{\prime}} \mathcal{C}\left(\mathbf{r}_{\|}, z, z^{\prime}, t\right)\right|_{z=z^{\prime}=0}
$$

in the bulk as well as in a film. Moreover, for $\alpha \in\{1, \ldots, d-$ $1\}$, one has

$$
\begin{aligned}
& \left\langle\left[\partial_{\alpha} \phi\left(\mathbf{r}_{\|}, z, r\right)\right]^{2}\right\rangle=-\left\langle\phi\left(\mathbf{r}_{\|}, z, t\right) \partial_{\alpha}^{2} \phi\left(\mathbf{r}_{\|}, z, t\right)\right\rangle \\
& \quad=-\left.\partial_{\alpha}^{2} \mathcal{C}\left(\mathbf{r}_{\|}, z, z, t\right)\right|_{\mathbf{r}_{\|}=\mathbf{0}},
\end{aligned}
$$

where we used translational invariance in the lateral directions in order to obtain the last equation. 


\section{FLUCTUATIONS OF THE CCF}

In equilibrium, the two-time correlation function of the instantaneous CCF follows from Eq. (2.18) and it is given by

$$
\left\langle\mathcal{K}(z, t) \mathcal{K}\left(z^{\prime}, t^{\prime}\right)\right\rangle=\left\langle\mathcal{P}_{f}(z, t) \mathcal{P}_{f}\left(z^{\prime}, t^{\prime}\right)\right\rangle-\left\langle\mathcal{P}_{f}(z)\right\rangle\left\langle\mathcal{P}_{b}\left(z^{\prime}\right)\right\rangle-\left\langle\mathcal{P}_{b}(z)\right\rangle\left\langle\mathcal{P}_{f}\left(z^{\prime}\right)\right\rangle+\left\langle\mathcal{P}_{b}(z, t) \mathcal{P}_{b}\left(z^{\prime}, t^{\prime}\right)\right\rangle,
$$

where we used the fact that film and bulk pressure are uncorrelated,

$$
\left\langle\mathcal{P}_{f}(z, t) \mathcal{P}_{b}\left(z^{\prime}, t^{\prime}\right)\right\rangle=\left\langle\mathcal{P}_{f}(z, t)\right\rangle\left\langle\mathcal{P}_{b}\left(z^{\prime}, t^{\prime}\right)\right\rangle
$$

since they emerge from physically separated parts of the system. Accordingly, the fluctuations of the CCF (per area) given by

$$
\Delta \mathcal{K}(z, t) \equiv \mathcal{K}(z, t)-\langle\mathcal{K}(z)\rangle
$$

are correlated in equilibrium as

$$
\begin{aligned}
\left\langle\Delta \mathcal{K}(z, t) \Delta \mathcal{K}\left(z^{\prime}, t^{\prime}\right)\right\rangle & =\left\langle\mathcal{K}(z, t) \mathcal{K}\left(z^{\prime}, t^{\prime}\right)\right\rangle-\langle\mathcal{K}(z)\rangle\left\langle\mathcal{K}\left(z^{\prime}\right)\right\rangle \\
& =\left\langle\mathcal{P}_{f}(z, t) \mathcal{P}_{f}\left(z^{\prime}, t^{\prime}\right)\right\rangle+\left\langle\mathcal{P}_{b}(z, t) \mathcal{P}_{b}\left(z^{\prime}, t^{\prime}\right)\right\rangle-\left\langle\mathcal{P}_{f}(z)\right\rangle\left\langle\mathcal{P}_{f}\left(z^{\prime}\right)\right\rangle-\left\langle\mathcal{P}_{b}(z)\right\rangle\left\langle\mathcal{P}_{b}\left(z^{\prime}\right)\right\rangle \\
& =\left\langle\Delta \mathcal{P}_{f}(z, t) \Delta \mathcal{P}_{f}\left(z^{\prime}, t^{\prime}\right)\right\rangle+\left\langle\Delta \mathcal{P}_{b}(z, t) \Delta \mathcal{P}_{b}\left(z^{\prime}, t^{\prime}\right)\right\rangle
\end{aligned}
$$

with $\Delta \mathcal{P}(z, t) \equiv \mathcal{P}(z, t)-\langle\mathcal{P}(z, t)\rangle$ and where we used Eq. (3.1) as well as

$$
\langle\mathcal{K}(z)\rangle\left\langle\mathcal{K}\left(z^{\prime}\right)\right\rangle=\left\langle\mathcal{P}_{f}(z)\right\rangle\left\langle\mathcal{P}_{f}\left(z^{\prime}\right)\right\rangle-\left\langle\mathcal{P}_{f}(z)\right\rangle\left\langle\mathcal{P}_{b}\left(z^{\prime}\right)\right\rangle-\left\langle\mathcal{P}_{f}\left(z^{\prime}\right)\right\rangle\left\langle\mathcal{P}_{b}(z)\right\rangle+\left\langle\mathcal{P}_{b}(z)\right\rangle\left\langle\mathcal{P}_{b}\left(z^{\prime}\right)\right\rangle
$$

Equation (3.4) reflects the fact that the variance of a sum of two uncorrelated random processes [ $\mathcal{P}_{f}$ and $-\mathcal{P}_{b}$, see Eq. $\left.(2.18)\right]$ is the sum of the individual variances. For $t-t^{\prime} \rightarrow \infty, \mathcal{P}_{f}(z, t)$ and $\mathcal{P}_{f}\left(z^{\prime}, t^{\prime}\right)$ become uncorrelated in equilibrium, i.e., $\left\langle\mathcal{P}_{f}(z, t) \mathcal{P}_{f}\left(z^{\prime}, t^{\prime}\right)\right\rangle \rightarrow\left\langle\mathcal{P}_{f}(z)\right\rangle\left\langle\mathcal{P}_{f}\left(z^{\prime}\right)\right\rangle$; this applies analogously to $\mathcal{P}_{b}$. Accordingly, one has

$$
\left\langle\Delta \mathcal{K}(z, t) \Delta \mathcal{K}\left(z^{\prime}, t^{\prime}\right)\right\rangle \stackrel{t-t^{\prime} \rightarrow \infty}{=} 0
$$

Since we consider equilibrium dynamics, we can exploit time-translation invariance and henceforth set $t^{\prime}=0$.

\section{A. Pressure correlation function}

Equation (3.4) can be evaluated by using Eqs. (2.18)-(2.20) as well as the fact the four-point correlation function for a multivariate zero-mean Gaussian process $X_{n} \sim \phi$ or $X_{n} \sim \partial_{\beta} \phi$ is given by the following cumulant expansion:

$$
\left\langle X_{i} X_{j} X_{k} X_{l}\right\rangle=\left\langle X_{i} X_{j}\right\rangle\left\langle X_{k} X_{l}\right\rangle+\left\langle X_{i} X_{k}\right\rangle\left\langle X_{j} X_{l}\right\rangle+\left\langle X_{i} X_{l}\right\rangle\left\langle X_{j} X_{k}\right\rangle
$$

Furthermore, we use the fact that $\int_{A} d^{d-1} r_{\|} \int_{A} d^{d-1} r_{\|}^{\prime} \mathcal{C}\left(\mathbf{r}_{\|}-\mathbf{r}_{\|}^{\prime}\right)=A \int_{A} d^{d-1} r_{\|} \mathcal{C}\left(\mathbf{r}_{\|}\right)$, which follows upon applying the (volume preserving) coordinate transformation $\left(\mathbf{r}_{\|}, \mathbf{r}_{\|}^{\prime}\right) \mapsto\left(\mathbf{r}_{\|}-\mathbf{r}_{\|}^{\prime}, \mathbf{r}_{\|}^{\prime}\right)$ and by using translational invariance in the lateral directions. In order to illustrate the calculation of $\left\langle\mathcal{P}_{f}(z, t) \mathcal{P}_{f}\left(z^{\prime}, 0\right)\right\rangle$, we consider the term

$$
\begin{aligned}
& \frac{1}{4 A^{2}} \int_{A} d s_{z}\left(\mathbf{r}_{\|}\right) \int_{A} d s_{z}\left(\mathbf{r}_{\|}^{\prime}\right)\left\langle\left[\partial_{z} \phi\left(\mathbf{r}_{\|}, z, t\right)\right]^{2}\left[\partial_{z^{\prime}} \phi\left(\mathbf{r}_{\|}^{\prime}, z^{\prime}, 0\right)\right]^{2}\right\rangle \\
& \quad=\Pi+\frac{1}{2 A^{2}} \int_{A} d s_{z}\left(\mathbf{r}_{\|}\right) \int_{A} d s_{z}\left(\mathbf{r}_{\|}^{\prime}\right)\left\langle\partial_{z} \phi\left(\mathbf{r}_{\|}, z, t\right) \partial_{z^{\prime}} \phi\left(\mathbf{r}_{\|}^{\prime}, z^{\prime}, 0\right)\right\rangle^{2}=\Pi+\frac{1}{2 A} \int_{A} d s_{z}\left(\mathbf{R}_{\|}\right)\left[\partial_{z} \partial_{z^{\prime}} \mathcal{C}\left(\mathbf{R}_{\|}, z, z^{\prime}, t\right)\right]^{2}
\end{aligned}
$$

where $d s_{z}\left(\mathbf{r}_{\|}\right)$denotes an area element and

$$
\begin{aligned}
\Pi & \equiv \frac{1}{4 A^{2}} \int_{A} d s_{z}\left(\mathbf{r}_{\|}\right)\left\langle\left[\partial_{z} \phi\left(\mathbf{r}_{\|}, z, t\right)\right]^{2}\right\rangle \int_{A} d s_{z}\left(\mathbf{r}_{\|}^{\prime}\right)\left\langle\left[\partial_{z^{\prime}} \phi\left(\mathbf{r}_{\|}^{\prime}, z^{\prime}, t\right)\right]^{2}\right\rangle \\
& =\frac{1}{4}\left[\partial_{z} \partial_{z^{\prime}} \mathcal{C}_{\mathrm{st}}\left(\mathbf{0}, z, z^{\prime}\right)\right]_{z^{\prime} \rightarrow z}\left[\partial_{z} \partial_{z^{\prime}} \mathcal{C}_{\mathrm{st}}\left(\mathbf{0}, z, z^{\prime}\right)\right]_{z \rightarrow z^{\prime}}
\end{aligned}
$$

represents a contribution to $\left\langle\mathcal{P}_{f}(z)\right\rangle\left\langle\mathcal{P}_{f}\left(z^{\prime}\right)\right\rangle$, resulting from the first term on the right-hand side of Eq. (3.7). In equilibrium, all these contributions are time independent $\left(\mathcal{C}_{\text {st }}\right.$ denotes the static equilibrium correlation function, see Sec. II C), and they are subtracted upon obtaining the CCF fluctuations according to Eq. (3.4) [see also Eq. (3.19) below]. Altogether, we obtain

$$
\begin{aligned}
\left\langle\mathcal{P}_{f}(z, t) \mathcal{P}_{f}\left(z^{\prime}, 0\right)\right\rangle= & \left\langle\mathcal{P}_{f}(z)\right\rangle\left\langle\mathcal{P}_{f}\left(z^{\prime}\right)\right\rangle+\frac{1}{A} \int_{A} d^{d-1} r\left\{\frac{1}{2}\left(\partial_{z} \partial_{z^{\prime}} \mathcal{C}\right)^{2}+\frac{1}{2} \sum_{\alpha, \beta=1}^{d-1}\left(-\partial_{\alpha} \partial_{\beta} \mathcal{C}\right)^{2}\right. \\
& +\mathcal{C} \sum_{\alpha, \beta=1}^{d} \partial_{\alpha}^{2} \hat{\partial}_{\beta}^{2} \mathcal{C}+\left(\sum_{\alpha=1}^{d} \partial_{\alpha}^{2} \mathcal{C}\right)\left(\sum_{\alpha=1}^{d} \hat{\partial}_{\alpha}^{2} \mathcal{C}\right)-\frac{1}{2} \sum_{\alpha=1}^{d-1}\left[\left(\partial_{z} \partial_{\alpha} \mathcal{C}\right)^{2}+\left(\partial_{z^{\prime}} \partial_{\alpha} \mathcal{C}\right)^{2}\right]
\end{aligned}
$$




$$
\begin{aligned}
& -\left[\left(\partial_{z} \mathcal{C}\right) \partial_{z} \sum_{\alpha=1}^{d} \hat{\partial}_{\alpha}^{2} \mathcal{C}+\left(\partial_{z^{\prime}} \mathcal{C}\right) \partial_{z^{\prime}} \sum_{\alpha=1}^{d} \partial_{\alpha}^{2} \mathcal{C}\right]+\sum_{\alpha=1}^{d-1}\left(\partial_{\alpha} \mathcal{C}\right) \partial_{\alpha}\left(\sum_{\beta=1}^{d} \partial_{\beta}^{2} \mathcal{C}+\sum_{\beta=1}^{d} \hat{\partial}_{\beta}^{2} \mathcal{C}\right)+\frac{1}{2} \tau^{2} \mathcal{C}^{2} \\
& \left.+\frac{1}{2} \tau\left[\left(\partial_{z} \mathcal{C}\right)^{2}+\left(\partial_{z^{\prime}} \mathcal{C}\right)^{2}\right]-\tau\left(\sum_{\alpha=1}^{d-1} \partial_{\alpha} \mathcal{C}\right)^{2}-\tau \mathcal{C}\left(\sum_{\alpha=1}^{d} \partial_{\alpha}^{2} \mathcal{C}+\sum_{\alpha=1}^{d} \hat{\partial}_{\alpha}^{2} \mathcal{C}\right)\right\}
\end{aligned}
$$

where $\partial_{\gamma} \in\left\{\partial_{r_{1}}, \ldots, \partial_{r_{d-1}}, \partial_{z}\right\}$ and we define $\hat{\partial}_{\gamma} \in\left\{\partial_{r_{1}}, \ldots, \partial_{r_{d-1}}, \partial_{z^{\prime}}\right\}$, such that $\hat{\partial}_{\gamma}=\partial_{\gamma}=\partial_{r_{\gamma}}$ for $\gamma=1, \ldots, d-1$ and $\hat{\partial}_{d}=\partial_{z^{\prime}}$; for brevity, we suppress the arguments of $\mathcal{C}\left(\mathbf{r}, z, z^{\prime}, t\right)$ on the right-hand side. The same expression results for $\left\langle\mathcal{P}_{b}(z, t) \mathcal{P}_{b}\left(z^{\prime}, 0\right)\right\rangle$, but with $\mathcal{C}$ replaced by the bulk correlator $\mathcal{C}_{b}$. It is useful to note that the quantity $\left\langle\Delta \mathcal{P}_{f}(z, t) \Delta \mathcal{P}_{f}(z, 0)\right\rangle=$ $\left\langle\mathcal{P}_{f}(z, t) \mathcal{P}_{f}\left(z^{\prime}, 0\right)\right\rangle-\left\langle\mathcal{P}_{f}(z)\right\rangle\left\langle\mathcal{P}_{f}\left(z^{\prime}\right)\right\rangle$ has the length dimension of $A^{-1} L^{-(d+1)}$, which can be readily inferred from Eqs. (2.27), (2.30), and (3.10).

We now specialize Eq. (3.10) to various BCs in thin films and make use of the available spatial symmetries. For Neumann and Dirichlet BCs we will obtain expressions in terms of the correlation function for periodic BCs. The film pressure variances are evaluated further in Secs. III B and III C.

\section{Bulk and periodic BCs}

In the following, we jointly analyze the pressure both for the bulk system and for thin films with periodic BCs in the transverse direction. We note that translational invariance [see Eq. (2.27)] implies

$$
\left\langle\left[\partial_{z} \phi\left(\mathbf{r}_{\|}, z, t\right)\right]^{2}\right\rangle=-\left\langle\phi\left(\mathbf{r}_{\|}, z, t\right) \partial_{z}^{2} \phi\left(\mathbf{r}_{\|}, z, t\right)\right\rangle=-\left.\partial_{z}^{2} \mathcal{C}^{(\mathrm{p})}\left(\mathbf{r}_{\|}=\mathbf{0}, z, t\right)\right|_{z=0},
$$

with the analogous expression applying in the bulk. Upon using Eqs. (2.36) and (3.11), the mean equilibrium CCF for a film, with one surface at $z=0$ and with periodic BCs, follows from Eq. (2.21) [9]:

$$
\left\langle\mathcal{K}^{(\mathrm{p})}\right\rangle=-\left.\frac{3}{2} \partial_{z}^{2} \mathcal{C}_{\mathrm{st}}^{(\mathrm{p})}\left(\mathbf{r}_{\|}=\mathbf{0}, z\right)\right|_{z=0}-\left.\frac{1}{2} \sum_{\alpha=1}^{d-1} \partial_{\alpha}^{2} \mathcal{C}_{\mathrm{st}}^{(\mathrm{p})}\left(\mathbf{r}_{\|}, 0\right)\right|_{\mathbf{r}_{\|}=\mathbf{0}} .
$$

The film pressure correlator in Eq. (3.10) reduces to

$$
\begin{aligned}
\left\langle\mathcal{P}_{f}^{(\mathrm{p})}(z, t) \mathcal{P}_{f}^{(\mathrm{p})}\left(z^{\prime}, 0\right)\right\rangle= & \left\langle\mathcal{P}_{f}^{(\mathrm{p})}\right\rangle^{2}+\frac{1}{A} \int_{A} d^{d-1} r\left\{\frac{1}{2}\left(-\partial_{z}^{2} \mathcal{C}^{(\mathrm{p})}\right)^{2}+\frac{1}{2} \sum_{\alpha, \beta=1}^{d-1}\left(-\partial_{\alpha} \partial_{\beta} \mathcal{C}^{(\mathrm{p})}\right)^{2}\right. \\
& +\mathcal{C}^{(\mathrm{p})} \sum_{\alpha, \beta=1}^{d} \partial_{\alpha}^{2} \partial_{\beta}^{2} \mathcal{C}^{(\mathrm{p})}+\left(\sum_{\alpha=1}^{d} \partial_{\alpha}^{2} \mathcal{C}^{(\mathrm{p})}\right)^{2}-\sum_{\alpha=1}^{d-1}\left(\partial_{z} \partial_{\alpha} \mathcal{C}^{(\mathrm{p})}\right)^{2}-2\left(\partial_{z} \mathcal{C}^{(\mathrm{p})}\right) \partial_{z} \sum_{\alpha=1}^{d} \partial_{\alpha}^{2} \mathcal{C}^{(\mathrm{p})} \\
& \left.+2 \sum_{\alpha=1}^{d-1}\left(\partial_{\alpha} \mathcal{C}^{(\mathrm{p})}\right) \partial_{\alpha} \sum_{\beta=1}^{d} \partial_{\beta}^{2} \mathcal{C}^{(\mathrm{p})}+\frac{1}{2} \tau^{2}\left(\mathcal{C}^{(\mathrm{p})}\right)^{2}+\tau\left(\partial_{z} \mathcal{C}^{(\mathrm{p})}\right)^{2}-\tau\left(\sum_{\alpha=1}^{d-1} \partial_{\alpha} \mathcal{C}^{(\mathrm{p})}\right)^{2}-2 \tau \mathcal{C}^{(\mathrm{p})} \sum_{\alpha=1}^{d} \partial_{\alpha}^{2} \mathcal{C}^{(\mathrm{p})}\right\}
\end{aligned}
$$

where we again suppressed the arguments of $\mathcal{C}\left(\mathbf{r}, z-z^{\prime}, t\right)$ on the right-hand side. In the special case $z=z^{\prime}=0$, Eq. (3.13) can be simplified using Eq. (2.35):

$$
\begin{aligned}
\left\langle\mathcal{P}_{f}^{(\mathrm{p})}(z=0, t) \mathcal{P}_{f}^{(\mathrm{p})}\left(z^{\prime}=0,0\right)\right\rangle= & \left\langle\mathcal{P}_{f}^{(\mathrm{p})}\right\rangle^{2}+\frac{1}{A} \int_{A} d^{d-1} r\left\{\frac{1}{2}\left(-\partial_{z}^{2} \mathcal{C}^{(\mathrm{p})}\right)^{2}\right. \\
& +\frac{1}{2} \sum_{\alpha, \beta=1}^{d-1}\left(-\partial_{\alpha} \partial_{\beta} \mathcal{C}^{(\mathrm{p})}\right)^{2}+\mathcal{C}^{(\mathrm{p})} \sum_{\alpha, \beta=1}^{d} \partial_{\alpha}^{2} \partial_{\beta}^{2} \mathcal{C}^{(\mathrm{p})}+\left(\sum_{\alpha=1}^{d} \partial_{\alpha}^{2} \mathcal{C}^{(\mathrm{p})}\right)^{2}+2 \sum_{\alpha=1}^{d-1}\left(\partial_{\alpha} \mathcal{C}^{(\mathrm{p})}\right) \partial_{\alpha} \sum_{\beta=1}^{d} \partial_{\beta}^{2} \mathcal{C}^{(\mathrm{p})} \\
& \left.+\frac{1}{2} \tau^{2}\left(\mathcal{C}^{(\mathrm{p})}\right)^{2}-\tau\left(\sum_{\alpha=1}^{d-1} \partial_{\alpha} \mathcal{C}^{(\mathrm{p})}\right)^{2}-2 \tau \mathcal{C}^{(\mathrm{p})} \sum_{\alpha=1}^{d} \partial_{\alpha}^{2} \mathcal{C}^{(\mathrm{p})}\right\}_{z=z^{\prime}=0}
\end{aligned}
$$

For bulk pressure correlations $\left\langle\mathcal{P}_{b}(z, t) \mathcal{P}_{b}\left(z^{\prime}, 0\right)\right\rangle$, the same expressions as in Eqs. (3.13) and (3.14) apply, but with $\mathcal{C}^{(\mathrm{p})}$ replaced by the bulk correlation function $\mathcal{C}_{b}$. 


\section{Neumann BCs}

The pressure correlation function for a film with Neumann BCs is given by Eq. (3.10) with $\mathcal{C}$ replaced by $\mathcal{C}^{(\mathrm{N})}$ [see Eq. $\left.(2.32 \mathrm{~b})\right]$. In the special case $z=z^{\prime}=0$, the resulting expression can be simplified by noting that Eq. (2.32b) implies

$$
\begin{aligned}
& \left.\partial_{z^{\prime}} \mathcal{C}^{(\mathrm{N})}\left(\mathbf{r}_{\|}, z, z^{\prime}, t\right)\right|_{z=z^{\prime}=0}=0, \\
& \left.\partial_{z} \mathcal{C}^{(\mathrm{N})}\left(\mathbf{r}_{\|}, z, z^{\prime}, t\right)\right|_{z=z^{\prime}=0}=\left.2 \partial_{z} \mathcal{C}^{(\mathrm{p})}\left(\mathbf{r}_{\|}, z=0, t\right)\right|_{2 L}=0, \\
& \left.\partial_{\alpha}^{n} \mathcal{C}^{(\mathrm{N})}\left(\mathbf{r}_{\|}, z, z^{\prime}, t\right)\right|_{z=z^{\prime}=0}=\left.2 \partial_{\alpha}^{n} \mathcal{C}^{(\mathrm{p})}\left(\mathbf{r}_{\|}, 0, t\right)\right|_{2 L}, \quad \alpha \in\{1, \ldots, d-1\}, \\
& \left.\partial_{z}^{2} \mathcal{C}^{(\mathrm{N})}\left(\mathbf{r}_{\|}, z, z^{\prime}, t\right)\right|_{z=z^{\prime}=0}=\left.\partial_{z^{\prime}}^{2} \mathcal{C}^{(\mathrm{N})}\left(\mathbf{r}_{\|}, z, z^{\prime}, t\right)\right|_{z=z^{\prime}=0}=\left.2 \partial_{z}^{2} \mathcal{C}^{(\mathrm{p})}\left(\mathbf{r}_{\|}, z=0, t\right)\right|_{2 L} .
\end{aligned}
$$

Using these relations in Eq. (3.10) renders

$$
\begin{aligned}
\left\langle\mathcal{P}_{f}^{(\mathrm{N})}(z=0, t) \mathcal{P}_{f}^{(\mathrm{N})}\left(z^{\prime}=0,0\right)\right\rangle= & \left\langle\mathcal{P}_{f}^{(\mathrm{N})}\right\rangle^{2}+\frac{1}{A} \int_{A} d^{d-1} r\left\{2 \sum_{\alpha, \beta=1}^{d-1}\left(-\partial_{\alpha} \partial_{\beta} \mathcal{C}^{(\mathrm{p})}\right)^{2}\right. \\
& +4 \mathcal{C}^{(\mathrm{p})} \sum_{\alpha, \beta=1}^{d} \partial_{\alpha}^{2} \partial_{\beta}^{2} \mathcal{C}^{(\mathrm{p})}+4\left(\sum_{\alpha=1}^{d} \partial_{\alpha}^{2} \mathcal{C}^{(\mathrm{p})}\right)^{2}+8 \sum_{\alpha=1}^{d-1}\left(\partial_{\alpha} \mathcal{C}^{(\mathrm{p})}\right) \partial_{\alpha} \sum_{\beta=1}^{d} \partial_{\beta}^{2} \mathcal{C}^{(\mathrm{p})} \\
& \left.+2 \tau^{2}\left(\mathcal{C}^{(\mathrm{p})}\right)^{2}-4 \tau\left(\sum_{\alpha=1}^{d-1} \partial_{\alpha} \mathcal{C}^{(\mathrm{p})}\right)^{2}-8 \tau \mathcal{C}^{(\mathrm{p})} \sum_{\alpha=1}^{d} \partial_{\alpha}^{2} \mathcal{C}^{(\mathrm{p})}\right\}_{\substack{z=z^{\prime}=0, L \mapsto 2 L}},
\end{aligned}
$$

where, as indicated on the right-hand side, the correlation function $\mathcal{C}^{(\mathrm{p})}$ for periodic BCs has to be evaluated for a film thickness of $2 L$.

\section{Dirichlet BCs}

We begin by noting that Eq. (2.32c) implies

$$
\begin{aligned}
\mathcal{C}^{(\mathrm{D})}\left(\mathbf{r}_{\|}, z=0, z^{\prime}=0, t\right) & =0, \\
\left.\partial_{z^{\prime}} \mathcal{C}^{(\mathrm{D})}\left(\mathbf{r}_{\|}, z, z^{\prime}, t\right)\right|_{z=z^{\prime}=0} & =-\left.2 \partial_{z} \mathcal{C}^{(\mathrm{p})}\left(\mathbf{r}_{\|}, z=0, t\right)\right|_{2 L}=0, \\
\left.\partial_{z}^{n} \mathcal{C}^{(\mathrm{D})}\left(\mathbf{r}_{\|}, z, z^{\prime}, t\right)\right|_{z=z^{\prime}=0} & =0, \\
\left.\partial_{\alpha}^{n} \mathcal{C}^{(\mathrm{D})}\left(\mathbf{r}_{\|}, z, z^{\prime}, t\right)\right|_{z=z^{\prime}=0} & =0 \\
\left.\partial_{z^{\prime}}^{2} \mathcal{C}^{(\mathrm{D})}\left(\mathbf{r}_{\|}, z, z^{\prime}, t\right)\right|_{z=z^{\prime}=0} & =0, \\
\left.\partial_{z} \partial_{z} \mathcal{C}^{(\mathrm{D})}\left(\mathbf{r}_{\|}, z, z^{\prime}, t\right)\right|_{z=z^{\prime}=0} & =-\left.2 \partial_{z}^{2} \mathcal{C}^{(\mathrm{p})}\left(\mathbf{r}_{\|}, z=0, t\right)\right|_{2 L} .
\end{aligned}
$$

Using these relations to evaluate the film pressure correlator in Eq. (3.10) for $z=z^{\prime}=0$ renders

$$
\left\langle\mathcal{P}_{f}^{(\mathrm{D})}(z=0, t) \mathcal{P}_{f}^{(\mathrm{D})}\left(z^{\prime}=0,0\right)\right\rangle=\left\langle\mathcal{P}_{f}^{(\mathrm{D})}\right\rangle^{2}+\frac{1}{A} \int_{A} d^{d-1} r\left\{2\left[-\partial_{z}^{2} \mathcal{C}^{(\mathrm{p})}(\mathbf{r}, z, t)\right]^{2}\right\}_{\substack{z=0, L \mapsto 2 L}} .
$$

\section{B. Equilibrium variance}

The equilibrium variance of the CCF at a fixed boundary (located at $z=z^{\prime}=0$ ) follows by setting $t=t^{\prime}=0$ in Eq. (3.4), resulting in

$$
\left\langle\Delta \mathcal{K}^{2}\right\rangle=\left\langle\mathcal{K}^{2}\right\rangle-\langle\mathcal{K}\rangle^{2}=\left\langle\mathcal{P}_{f}^{2}\right\rangle-\left\langle\mathcal{P}_{f}\right\rangle^{2}+\left\langle\mathcal{P}_{b}^{2}\right\rangle-\left\langle\mathcal{P}_{b}\right\rangle^{2}=\left\langle\left(\Delta \mathcal{P}_{f}\right)^{2}\right\rangle+\left\langle\left(\Delta \mathcal{P}_{b}\right)^{2}\right\rangle
$$

This expression is evaluated in the following by replacing in Eq. (3.10) $\mathcal{C}$ by the expression of $\mathcal{C}_{\mathrm{st}}$ for the respective BCs. Below we discuss the final results at bulk criticality $(\tau=0)$.

\section{Bulk system}

The static equilibrium variance of the bulk pressure $\left\langle\Delta \mathcal{P}_{b}^{2}\right\rangle \equiv\left\langle\mathcal{P}_{b}^{2}\right\rangle-\left\langle\mathcal{P}_{b}\right\rangle^{2}$ is obtained by inserting the equilibrium bulk correlation function $\mathcal{C}_{\mathrm{b}, \mathrm{st}}$ [Eq. (2.23)] for $\mathcal{C}$ into Eq. (3.10). In order to simplify this calculation, we note that, due to the translational invariance of $\mathcal{C}_{b},\left\langle\Delta \mathcal{P}_{b}^{2}\right\rangle$ takes the same form as the variance for periodic BCs reported in Eq. (3.14) (with $\mathcal{C}^{\text {(p) }}$ replaced by $\mathcal{C}_{b}$ ). We furthermore recall the Schwinger-Dyson relation (see, e.g., Ref. [9])

$$
\left\langle\phi(\mathbf{r}) \frac{\delta \mathcal{F}}{\delta \phi\left(\mathbf{r}^{\prime}\right)}\right\rangle=\delta\left(\mathbf{r}-\mathbf{r}^{\prime}\right)
$$


which implies the following identity for the equilibrium bulk correlation function:

$$
-\nabla^{2} \mathcal{C}_{\mathrm{b}, \mathrm{st}}(\mathbf{r})+\tau \mathcal{C}_{\mathrm{b}, \mathrm{st}}(\mathbf{r})=\delta(\mathbf{r})
$$

Using this relation in Eq. (3.14) renders

$$
\begin{aligned}
\left\langle\Delta \mathcal{P}_{b}^{2}\right\rangle \equiv\left\langle\mathcal{P}_{b}^{2}\right\rangle-\left\langle\mathcal{P}_{b}\right\rangle^{2}= & \frac{1}{A}\left\{-\mathbf{n} \cdot\left[\mathcal{C}_{\mathrm{b}, \mathrm{st}} \nabla_{\|} \delta\left(\mathbf{r}_{\|}\right)+\delta\left(\mathbf{r}_{\|}\right) \nabla_{\|} \mathcal{C}_{\mathrm{b}, \mathrm{st}}\right]_{\partial A} \delta(z)-\mathcal{C}_{\mathrm{b}, \mathrm{st}}\left(\mathbf{0}_{\|}, z\right) \partial_{z}^{2} \delta(z)-\delta(z) \partial_{z}^{2} \mathcal{C}_{\mathrm{b}, \mathrm{st}}\left(\mathbf{0}_{\|}, z\right)\right\}_{z=0} \\
& +\frac{1}{A} \int_{A} d^{d-1} r\left\{\frac{1}{2}\left[-\partial_{z}^{2} \mathcal{C}_{\mathrm{b}, \mathrm{st}}\right]^{2}+\frac{1}{2} \sum_{\alpha, \alpha^{\prime}}^{d-1}\left[-\partial_{\alpha} \partial_{\alpha^{\prime}} \mathcal{C}_{\mathrm{b}, \mathrm{st}}\right]^{2}+\frac{1}{2} \tau^{2} \mathcal{C}_{\mathrm{b}, \mathrm{st}}^{2}+\tau\left[\nabla_{\|} \mathcal{C}_{\mathrm{b}, \mathrm{st}}\right]^{2}\right\}_{z=0},
\end{aligned}
$$

where $\mathbf{n}$ denotes the unit normal of the $(d-1)$-dimensional surface plane.

Upon inserting $\mathcal{C}_{\mathrm{b}, \mathrm{st}}$ [Eq. (2.23)] into Eq. (3.22), one finds that the resulting integral over $A$ generally converges at its upper limit of large distances $r$ (where $r$ refers to the spherical coordinate), allowing one to set $A \rightarrow \infty$ for its evaluation. However, the integral does not converge at the lower limit $r \rightarrow 0$, requiring a regularization via a small-distance cutoff $\varepsilon$. Similarly, a regularization is also necessary for the terms in the first line of Eq. (3.22). To this end, we evaluate their Fourier space representation by taking into account a wave-number cutoff $q_{\max } \simeq 1 / \varepsilon$ and, accordingly, regularize the $\delta$ function as [16] $\delta(z)=\int_{-1 / \varepsilon}^{1 / \varepsilon} \frac{d q}{2 \pi} \exp (i q z)$, which results in

$$
\left.\delta(0)\right|_{\mathrm{reg}}=\frac{1}{\pi \varepsilon},\left.\quad \partial_{z}^{2} \delta(z=0)\right|_{\mathrm{reg}}=-\frac{1}{3 \pi \varepsilon^{3}} .
$$

Analogously, based on the Fourier representation in Eq. (2.22), we obtain the regularized form of the bulk correlator (at $\tau=0$ )

$$
\begin{aligned}
\left.\mathcal{C}_{\mathrm{b}, \mathrm{st}}\left(\mathbf{0}_{\|}, 0\right)\right|_{\mathrm{reg}} & =\frac{2}{(4 \pi)^{d / 2}(d-2) \Gamma(d / 2)} \frac{1}{\varepsilon^{d-2}}, \\
\left.\partial_{z}^{2} \mathcal{C}_{\mathrm{b}, \mathrm{st}}\left(\mathbf{0}_{\|}, z=0\right)\right|_{\mathrm{reg}} & =-\frac{1}{(4 \pi)^{d / 2} d \Gamma((d+1) / 2)} \frac{1}{\varepsilon^{d}} .
\end{aligned}
$$

The term $-\mathbf{n} \cdot[\ldots]_{\partial A} \delta(z)$ in Eq. (3.22) vanishes because the boundary $\partial A$ of the surface is assumed to be located basically at infinity, such that $r_{\|, \alpha} \neq 0$.

Altogether, the variance of the bulk pressure at bulk criticality $(\tau=0)$ turns out to be

$$
\left\langle\Delta \mathcal{P}_{b}^{2}\right\rangle \equiv\left\langle\mathcal{P}_{b}^{2}\right\rangle-\left\langle\mathcal{P}_{b}\right\rangle^{2}=\left[\frac{d(d-1) \Gamma(d / 2)^{2}}{4 \pi^{(d+1) / 2}(d+1) \Gamma((d-1) / 2)}+\frac{\left(d^{2}+3 d-6\right)}{3 \times 2^{d} \pi^{d / 2+1} d(d-2) \Gamma(1+d / 2)}\right] \frac{1}{A \varepsilon^{d+1}} .
$$

This expression is singular at $d=2$ and positive for all other integer dimensions $d$.

\section{Periodic BCS}

According to Eq. (2.32a), the static OP correlator for a thin film with periodic BCs follows as

$$
\begin{aligned}
\mathcal{C}_{\mathrm{st}}^{(\mathrm{p})}\left(r_{\|}, z\right) & =\sum_{m=-\infty}^{\infty} \mathcal{C}_{\mathrm{b}, \mathrm{st}}\left(\left\{r_{\|}, z+m L\right\}\right)=\frac{\Gamma(d / 2-1)}{4 \pi^{d / 2}} \sum_{m=-\infty}^{\infty} \frac{1}{\left|(z+m L)^{2}+r_{\|}^{2}\right|^{(d-2) / 2}} \\
& =\frac{\Gamma(d / 2-1)}{4 \pi^{d / 2} L^{d-2}} \sum_{m=-\infty}^{\infty} \frac{1}{\left|(\hat{z}+m)^{2}+\hat{r}_{\|}^{2}\right|^{(d-2) / 2}} \equiv L^{2-d} \hat{\mathcal{C}}_{\mathrm{st}}^{(\mathrm{p})}\left(\hat{r}_{\|}, \hat{z}\right),
\end{aligned}
$$

where $\mathcal{C}_{\mathrm{b}, \mathrm{st}}(r)$ denotes the bulk correlation function [Eq. (2.23)]. In the last line we introduced the dimensionless coordinates $\hat{z} \equiv z / L$ and $\hat{r}_{\|} \equiv r_{\|} / L$ and defined the scaling function $\hat{\mathcal{C}}_{\mathrm{st}}^{(\mathrm{p})}$. We note that in Eq. (3.26) the bulk correlation function corresponds to the term with $m=0$.

Using Eq. (2.32a) in Eq. (3.21) yields the following identity for the film correlation function:

$$
-\nabla^{2} \mathcal{C}_{\mathrm{st}}^{(\mathrm{p})}\left(\mathbf{r}_{\|}, z\right)+\tau \mathcal{C}_{\mathrm{st}}^{(\mathrm{p})}\left(\mathbf{r}_{\|}, z\right)=\delta\left(\mathbf{r}_{\|}\right) \sum_{m=-\infty}^{\infty} \delta(z+m L) .
$$

Using this relation in Eq. (3.13) with $z=z^{\prime}=0$ renders for $\left\langle\left(\Delta \mathcal{P}_{f}^{(\mathrm{p})}\right)^{2}\right\rangle$ the same form as in Eq. (3.22), with $\mathcal{C}_{\mathrm{b}, \mathrm{st}}$ replaced by $\mathcal{C}_{\mathrm{st}}^{(\mathrm{p})}$. Furthermore, upon inserting Eq. (3.26) into the resulting expression for $\left\langle\left(\Delta \mathcal{P}_{f}^{(\mathrm{p})}\right)^{2}\right\rangle$ and using the relation $\partial_{\beta} \mathcal{C}_{\mathrm{st}}\left(\mathbf{r}_{\|}, z\right)=L^{-1} \partial_{\hat{\beta}} \hat{\mathcal{C}}_{\mathrm{st}}\left(\hat{r}_{\|}, \hat{z}\right)$, the integral over the transverse area $A$ converges for $m \neq 0$ and results in expressions of the form $\sum_{m_{1}=1}^{\infty} \sum_{m_{2}=1}^{\infty}\left(m_{1}+m_{2}\right)^{-d-1}$, which can be evaluated in terms of the Riemann zeta function $\zeta$. For $m_{1}=0$ or $m_{2}=0$, the integral over $A$ does not converge at its lower limit $\left(r_{\|} \rightarrow 0\right)$ and is thus evaluated using a small-distance cutoff $\varepsilon$ for the radial 
coordinate $r_{\|}$. The integral over $A$ generally converges at its upper (large-distance) limit. Altogether, we obtain the following film pressure variance for periodic BCs with $\tau=0$ :

$$
\left\langle\left(\Delta \mathcal{P}_{f}^{(\mathrm{p})}\right)^{2}\right\rangle=\left\langle\mathcal{P}_{f}^{2}\right\rangle-\left\langle\mathcal{P}_{f}\right\rangle^{2}=\frac{1}{A L^{d+1}}\left[d(d-1) \pi^{-d / 2} \Gamma(d / 2) \zeta(d)-\frac{d \Gamma(d / 2)^{2} \zeta(d)}{\pi^{(d+1) / 2} \Gamma((d-1) / 2)} \frac{L}{\varepsilon}\right]+\left\langle\Delta \mathcal{P}_{b}^{2}\right\rangle .
$$

The power laws for $L$ and $\varepsilon$ emerge by using the scaling form for $\mathcal{C}_{\mathrm{b}, \mathrm{st}}$ given in Eq. (3.26). It turns out that $\left\langle\left(\Delta \mathcal{P}_{f}^{(\mathrm{p})}\right)^{2}\right\rangle A L^{d+1}$, which is only a function of $d$ and the dimensionless parameter $L / \varepsilon$, remains positive for all $d>2$ and all $L / \varepsilon>0$. In the relevant regime $L / \varepsilon \gg 1$, the variance $\left\langle\left(\Delta \mathcal{P}_{f}^{(\mathrm{p})}\right)^{2}\right\rangle$ is, in fact, dominated by $\left\langle\Delta \mathcal{P}_{b}^{2}\right\rangle \propto 1 /\left(A \varepsilon^{d+1}\right)$ [see Eq. (3.25)].

\section{Neumann BCs}

Equation (3.16) renders, after some algebra analogous to the one leading to Eq. (3.28), the static variance

$$
\begin{aligned}
\left\langle\left(\Delta \mathcal{P}_{f}^{(\mathrm{N})}\right)^{2}\right\rangle= & \left\langle\left(\mathcal{P}_{f}^{(\mathrm{N})}\right)^{2}\right\rangle-\left\langle\mathcal{P}_{f}^{(\mathrm{N})}\right\rangle^{2}=\frac{1}{A L^{d+1}}\left[\frac{d(d-1) \Gamma(d / 2) \zeta(d)}{(4 \pi)^{d / 2}}-\frac{\Gamma(d / 2)^{2} \zeta(d)}{2^{d-2} \pi^{(d+1) / 2} \Gamma((d-1) / 2)} \frac{L}{\varepsilon}\right] \\
& +\frac{1}{A \varepsilon^{d+1}}\left[\frac{\left(d^{2}-d-1\right) \Gamma(d / 2)^{2}}{\pi^{(d+1) / 2}(d+1) \Gamma((d-1) / 2)}+\frac{4\left(d^{2}+3 d-6\right)}{3 \times 2^{d} \pi^{d / 2+1} d(d-2) \Gamma(1+d / 2)}\right],
\end{aligned}
$$

where $\varepsilon$ denotes a small length scale required for regularizing the integral over the surface area. The term $\propto 1 /\left(A \varepsilon^{d+1}\right)$ scales like the bulk pressure variance [see Eq. (3.25)], but its detailed form differs from the one in Eq. (3.28) due to the different structures of Eqs. (3.14) and (3.16).

\section{Dirichlet BCS}

Upon introducing, as above, a small-distance cutoff $\varepsilon$ at the lower limit of the areal integral in Eq. (3.18), the static pressure variance follows as ${ }^{6}$

$$
\begin{aligned}
\left\langle\left(\Delta \mathcal{P}_{f}^{(\mathrm{D})}\right)^{2}\right\rangle=\left\langle\left(\mathcal{P}_{f}^{(\mathrm{D})}\right)^{2}\right\rangle-\left\langle\mathcal{P}_{f}^{(\mathrm{D})}\right\rangle^{2}= & \frac{1}{A L^{d+1}}\left[\frac{d(d-1) \Gamma(d / 2) \zeta(d)}{(4 \pi)^{d / 2}}-\frac{(d-1) \Gamma(d / 2)^{2} \zeta(d)}{2^{d-2} \pi^{(d+1) / 2} \Gamma((d-1) / 2)} \frac{L}{\varepsilon}\right] \\
& +\frac{1}{A \varepsilon^{d+1}} \frac{\Gamma(d / 2)^{2}}{2 \pi^{(d+1) / 2}(d+1) \Gamma((d-1) / 2)} .
\end{aligned}
$$

The universal subdominant term (first term in the square brackets) coincides with Eq. (14) in Ref. [16], as does the dominant (bulklike) scaling behavior for small $\varepsilon$ [given by the last term in Eq. (3.30) and by Eq. (13) in Ref. [16]].

\section{Discussion}

According to Eq. (3.19), the variance of the CCF (per area $A$ and thermal energy $k_{B} T$ ) is given by the sum of the bulk and film pressure variances. The film and bulk pressure variances determined above [see Eqs. (3.25) and (3.28)-(3.30)] pertain to a thin film geometry $\left(A=L_{\|}^{d-1} \rightarrow \infty\right)$ and thus represent the leading terms in an expansion in terms of $1 / A$ (for $\varepsilon$ nonzero). We thus expect the above results to represent a reasonable estimate for systems with a sufficiently large aspect ratio $L_{\|} / L$, such that the lateral OP modes approximately form a continuum spectrum. Thus, in the limit of small $\varepsilon \rightarrow 0$, the static equilibrium variance of the CCF turns out to be dominated by bulklike contributions, which induce the scaling behavior

$$
\left\langle(\Delta \mathcal{K})^{2}\right\rangle^{1 / 2} \propto \frac{1}{A^{1 / 2} \varepsilon^{(d+1) / 2}},
$$

where the proportionality constant is of $O(1)$ [see Eqs. (3.28)-(3.30)]. We emphasize that Eq. (3.31) quantifies the typical fluctuations of the Casimir pressure $\mathcal{K}$. Those of the actual Casimir force acting on a surface of area $A$ are characterized by $A\left\langle(\Delta \mathcal{K})^{2}\right\rangle^{1 / 2}$ and thus exhibit the expected thermodynamic scaling behavior [31]. The essential scaling behavior expressed in Eq. (3.31) has been confirmed by Monte Carlo simulations of various lattice models (see Refs. [17] $]^{7}$ ). Compared to the mean values of the CCF [40],

$$
\begin{aligned}
\left\langle\mathcal{K}^{(\mathrm{p})}\right\rangle & =L^{-d} \pi^{-d / 2} \Gamma(d / 2)(1-d) \zeta(d) \stackrel{d=3}{\simeq}-\frac{0.38}{L^{3}}, \\
\left\langle\mathcal{K}^{(\mathrm{N})}\right\rangle & =2^{-d}\left\langle\mathcal{K}^{(\mathrm{p})}\right\rangle, \\
\left\langle\mathcal{K}^{(\mathrm{D})}\right\rangle & =2^{-d}\left\langle\mathcal{K}^{(\mathrm{p})}\right\rangle,
\end{aligned}
$$

\footnotetext{
${ }^{6}$ The singular terms in the first line in Eq. (3.22) vanish for Dirichlet BCs in a cutoff-regularized field theory [see Eq. (3.17)] and we thus neglected them here.

${ }^{7}$ In Ref. [17], a different definition of the variance has been used, leading to slightly different geometric factors compared to Eq. (3.31) and Ref. [16].
} 
the dispersion $\left\langle(\Delta \mathcal{K})^{2}\right\rangle^{1 / 2}$ of the CCF is orders of magnitude larger and nonuniversal, as noted previously in Ref. [16]. In order to address this issue, it has been argued in Ref. [16] that the static variance is unobservable because any measurement device experiences a force averaged over a finite time interval. The variance of the average force $\overline{\mathcal{K}}$ is thus reduced by a factor $N=t_{\text {res }} / t_{\text {corr }}$, where $t_{\text {res }}$ is the temporal resolution of the measurement device and $t_{\text {corr }}$ is the correlation time of short-wavelength fluctuations (which provide the dominant contribution to the variance). For typical experimental setups, $N$ is estimated to be of $O\left(10^{4}\right)$, which reduces the dispersion $\left\langle\Delta \overline{\mathcal{K}}^{2}\right\rangle^{1 / 2}=N^{-1 / 2}\left\langle\Delta \mathcal{K}^{2}\right\rangle^{1 / 2}$ to a value comparable to the mean force $\langle\mathcal{K}\rangle[16]$.

In the remaining part of this study, we shall analyze temporal pressure correlations as well as experimentally observable quantities influenced by the fluctuations of the CCF. We find that in these cases the cutoff dependence is mitigated or even disappears, without the need to invoke the temporal resolution of the measurement device and to formulate specific assumptions about it.

\section{Dynamic pressure correlations}

We first focus on a film with periodic BCs. The associated two-time correlation function of the CCF at a fixed surface $\left(z=z^{\prime}=0\right)$ results from Eq. (3.4) as

$$
\langle\Delta \mathcal{K}(t) \Delta \mathcal{K}(0)\rangle=\left\langle\Delta \mathcal{P}_{b}(t) \Delta \mathcal{P}_{b}(0)\right\rangle+\left\langle\Delta \mathcal{P}_{f}^{(\mathrm{p})}(t) \Delta \mathcal{P}_{f}^{(\mathrm{p})}(0)\right\rangle
$$

In the following, we evaluate the dynamic bulk and film pressure correlations based on the formalism developed in Secs. III A 1 and III A 2.

\section{Bulk pressure correlations}

In the short-time limit $t \ll \tau^{-2}$ (which includes the critical case $\tau=0$ ), terms proportional to $\tau$ or $\tau^{2}$ in Eq. (3.10) are subdominant and can be asymptotically neglected. ${ }^{8}$ Accordingly, in this regime, the dynamic bulk pressure correlations at a surface located at $z=0$ are obtained by inserting Eq. (2.26) into Eq. (3.14):

$$
\left.\left\langle\Delta \mathcal{P}_{b}(t) \Delta \mathcal{P}_{b}(0)\right\rangle\right|_{t \ll \tau^{-2}}=\left\langle\mathcal{P}_{b}(t) \mathcal{P}_{b}(0)\right\rangle-\left\langle\mathcal{P}_{b}\right\rangle^{2}=\frac{H_{d}}{A} t^{-(d+1) / 4}, \quad \text { with } \quad H_{d} \equiv \Omega_{d-1} \int_{0}^{\infty} d \psi F_{d}(\psi),
$$

where

$$
\Omega_{d}=\frac{2 \pi^{d / 2}}{\Gamma(d / 2)}
$$

is the surface area of the $d$-dimensional unit sphere and $F_{d}(\psi)$ is a time-independent function which is constant for $\psi \rightarrow 0$ and $d>2$, while it vanishes exponentially for $\psi \rightarrow \infty$. Its explicit form is rather lengthy and thus it is not reported here. A numerical evaluation of $H_{d}$, which is finite in spatial dimensions $d>2$, gives $H_{3} \simeq 5.299 \times 10^{-3}$ and $H_{4} \simeq 5.924 \times 10^{-4}$.

Conversely, at long times $t \gg \tau^{-2}$, using the scaling form given in Eq. (2.25) for Eq. (3.14), the bulk pressure correlations are dominated by the term $(1 / 2) \tau^{2} \mathcal{C}\left(\mathbf{r}_{\|}, 0, t\right)^{2}$. Evaluating the remaining integral over $\mathbf{r}_{\|}$renders

$$
\left.\left\langle\Delta \mathcal{P}_{b}(t) \Delta \mathcal{P}_{b}(0)\right\rangle\right|_{t \gg \tau^{-2}}=\frac{1}{2^{3(d+1) / 2} \pi^{(d+1) / 2} A}(\tau t)^{-(d+1) / 2} .
$$

\section{Periodic BCs}

In order to determine the dynamic correlations of the film pressure at a fixed surface $(z=0)$, we insert Eq. (2.27) into Eq. (3.14). A typical term is given by, e.g.,

$$
\begin{aligned}
\frac{1}{A} & \int d^{d-1} r\left[\partial_{\alpha}^{2} \mathcal{C}^{(\mathrm{p})}\left(\mathbf{r}, z-z^{\prime}, t\right)\right]\left[\partial_{\beta}^{2} \mathcal{C}^{(\mathrm{p})}\left(\mathbf{r}, z-z^{\prime}, t\right)\right]_{z=z^{\prime}} \\
& =\left.\frac{1}{A L^{2}} \int d^{d-1} r \sum_{m, n=-\infty}^{\infty} \int \frac{d^{d-1} p}{(2 \pi)^{d-1}} \int \frac{d^{d-1} \tilde{p}}{(2 \pi)^{d-1}} p_{\alpha}^{2} \tilde{p}_{\beta}^{2} e^{i(\mathbf{p}+\tilde{\mathbf{p}}) \cdot \mathbf{r}+i\left(z-z^{\prime}\right) k_{m}-i\left(z-z^{\prime}\right) k_{n}} S\left(p, k_{m}, t\right) S\left(\tilde{p}, k_{n}, t\right)\right|_{z-z^{\prime}=0} \\
& =\frac{1}{A L^{2}} \sum_{m, n=-\infty}^{\infty} \int \frac{d^{d-1} p}{(2 \pi)^{d-1}} p_{\alpha}^{2} p_{\beta}^{2} S\left(p, k_{m}, t\right) S\left(p, k_{n}, t\right)
\end{aligned}
$$

where $S(p, k, t)$ is reported in Eq. (2.29). In summary, the dynamic film pressure correlation at a fixed surface is given by

$$
\begin{aligned}
\left\langle\Delta \mathcal{P}_{f}^{(\mathrm{p})}(t) \Delta \mathcal{P}_{f}^{(\mathrm{p})}(0)\right\rangle & =\left\langle\mathcal{P}_{f}^{(\mathrm{p})}(t) \mathcal{P}_{f}^{(\mathrm{p})}(0)\right\rangle-\left\langle\mathcal{P}_{f}^{(\mathrm{p})}\right\rangle^{2} \\
& =\frac{K_{d-1}}{2 A L^{2}} \sum_{m, n=-\infty}^{\infty} \int_{0}^{\infty} d p S\left(p, k_{m}, t\right) S\left(p, k_{n}, t\right) p^{d-2}\left[k_{m}^{2} k_{n}^{2}+2\left(p^{2}+k_{n}^{2}\right)\left(k_{m}^{2}+k_{n}^{2}\right)+p^{4}+2 \tau\left(2 k_{n}^{2}+p^{2}\right)+\tau^{2}\right]
\end{aligned}
$$

with $K_{d} \equiv \Omega_{d} /(2 \pi)^{d}$ and $\Omega_{d}$ reported in Eq. (3.35).

${ }^{8}$ This can be shown by inserting the critical bulk correlator $\mathcal{C}_{b}$ given in Eq. (2.26) into Eq. (3.14). 
The integrand $\mathcal{I}\left(p, k_{m}, k_{n}\right)$ in Eq. (3.38) has the symmetry $\mathcal{I}\left(p, k_{m}, k_{n}\right)=\mathcal{I}\left(p,-k_{m},-k_{n}\right)$. While it is not symmetric upon exchanging $k_{m}$ and $k_{n}$, it can be written in such a form by using the fact that $m$ and $n$ are summed over. (In the following discussion, $k_{m}$ and $k_{n}$ refer to the second and third argument of $\mathcal{I}$, respectively.) For small $p$ and $\tau=0$, the integrand behaves as $\mathcal{I} \propto p^{d-2}$ if $k_{m} \neq 0$ as well as if $k_{m}=k_{n}=0$, while $\mathcal{I} \propto p^{d-4}$ if $k_{m}=0, k_{n} \neq 0$; for $\tau \neq 0$ one has $\mathcal{I} \propto p^{d-2}$ at small $p$. Accordingly, for $\tau=0$ and $d \leqslant 3$, the integral in Eq. (3.38) is infrared divergent. This divergence is cut off by a finite lateral extent of the confining surfaces. For $t=0$, the integrand diverges as $\mathcal{I} \propto p^{d-2}$ for large $p$, which reflects the divergence of the static equilibrium variance [see Eq. (3.28)]. Owing to the exponential factor in $S(p, k, t)$, the sums and the integral in Eq. (3.38) are rapidly converging for large momenta $p$ and $k$ and $t>0$.

We now proceed by analyzing Eq. (3.38) for $d>2$ in various asymptotic limits, in which exact analytical expressions can be obtained. In general, the asymptotic behavior is controlled by the exponentials in Eq. (3.38) [see Eq. (2.29)], such that the integrand contributes significantly only if $q^{4} t+q^{2} \tau t \lesssim 1$, where $q^{2} \equiv p^{2}+k_{m}^{2}$. This requires $q \lesssim t^{-1 / 4}$ and $q \lesssim(\tau t)^{-1 / 2}$. Analogously to Eq. (2.24), one can identify two characteristic temporal regimes, depending on which of the two terms dominates. We analyze them separately:

Case $t \ll \tau^{-2}$, which includes $\tau=0$. Upon inserting Eq. (2.33) into Eq. (3.13), one readily infers that in this regime contributions $\propto \tau$ or $\tau^{2}$ to the pressure correlator are subdominant. A characteristic long-time behavior emerges for $t \gg L^{4}$, provided that also $\tau^{-2} \gg L^{4}$ holds, i.e., $L^{4} \ll t \ll \tau^{-2}$. In this regime, the dominant contribution to the integrand in Eq. (3.38) stems from small wave numbers, for which $p \ll t^{-1 / 4}, k_{j} \ll t^{-1 / 4}$. Thus, setting $k_{m}=k_{n}=0$, in Eq. (3.38) all terms involving $\tau$ vanish and the integral can be readily evaluated, providing the long-time asymptotic behavior (valid for $d>2$ )

$$
\left\langle\Delta \mathcal{P}_{f}^{(\mathrm{p})}\left(L^{4} \ll t \ll \tau^{-2}\right) \Delta \mathcal{P}_{f}^{(\mathrm{p})}(0)\right\rangle \simeq \frac{1}{2^{(7 d-3) / 4} \pi^{(d-1) / 2} \Gamma(d / 4+1 / 4) A L^{2}} t^{-(d-1) / 4} .
$$

The difference between Eq. (3.39) and the corresponding scaling behavior in the bulk [Eq. (3.34)] stems from the continuum spectrum of modes with small $k$ associated with the infinite transverse $(z)$ direction of the bulk system. We finally remark that, in a completely finite volume, one expects a long-time relaxation behavior different from Eq. (3.39) due to the isolated zero mode, which is absent for conserved dynamics [9].

At short times ( $t \ll L^{4}$, still keeping $t \ll \tau^{-2}$ ), instead, modes with $k_{j} \lesssim t^{-1 / 4}$ contribute significantly to the integral in Eq. (3.38). We first focus on dimensions $d \geqslant 4$, in which case the integrand in Eq. (3.38) is typically finite for $p \rightarrow 0$. In order to estimate $\left\langle\Delta \mathcal{P}_{f}^{(\mathrm{p})}(t) \Delta \mathcal{P}_{f}^{(\mathrm{p})}(0)\right\rangle$ at short times, we replace the sum over the modes by an integral using $\sum_{n} f\left(k_{n}\right)=$ $(L /(2 \pi)) \int d k f(k)$. According to Eq. (3.13), this replacement can be equivalently performed in the film correlation function in Eq. (2.27), rendering [see also Eq. (2.24)] $\left.\left.\mathcal{C}^{(\mathrm{p})}\left(\mathbf{r}_{\|}, z-z^{\prime}, t \ll L^{4}\right)\right|_{t \ll \tau^{-2}} \simeq \mathcal{C}_{b}\left(\mathbf{r}_{\|}, z-z^{\prime}, t\right)\right|_{\tau=0}$ in terms of the bulk correlation function given in Eq. (2.26). Thus, at short times and for reduced temperatures $\tau$ close to bulk criticality, the film and bulk pressure correlations [see Eq. (3.34)] essentially coincide, i.e.,

$$
\left.\left\langle\Delta \mathcal{P}_{f}^{(\mathrm{p})}\left(t \ll \min \left(L^{4}, \tau^{-2}\right)\right) \Delta \mathcal{P}_{f}^{(\mathrm{p})}(0)\right\rangle\right|_{d \geqslant 4} \simeq\left\langle\Delta \mathcal{P}_{b}(t) \Delta \mathcal{P}_{b}(0)\right\rangle=\frac{H_{d}}{A} t^{-(d+1) / 4} .
$$

Note that the notions of a short- and long-time limit are different in the film and in the bulk (compare Sec. III C 1).

For dimensions $d<4$, the contribution to the integrand in Eq. (3.38) pertaining to $k_{m}=0, k_{n} \neq 0$ is diverging for $p \rightarrow 0$ (as discussed above) and thus has to be analyzed separately in order to properly determine the short-time behavior. The scaling behavior of this contribution is found to be

$$
p=\frac{t^{-d / 4} K_{d-1}}{4 \pi A L} \int_{\lambda t^{1 / 4}}^{\infty} d P \int_{-\infty}^{\infty} d K P^{d-4} \frac{P^{4}+2 K^{2} P^{2}+2 K^{4}}{K^{2}+P^{2}} e^{-K^{4}-2 K^{2} P^{2}-2 P^{4}}=\frac{t^{-d / 4} K_{d-1}}{4 \pi A L}\left[\Gamma(3 / 4) \int_{\lambda t^{1 / 4}}^{\sigma} d P P^{d-4}+\mathcal{R}(\sigma)\right],
$$

where $\sigma \lesssim 1$ is an arbitrary dimensionless parameter introduced to enable the small- $P$ expansion of the integrand, and $R(\sigma)$ is a remainder, which is exponentially suppressed and will be omitted henceforth. Since the first equation is independent of $\sigma$, the sum in the second equation must be independent of $\sigma$, too. The required small momentum cutoff $\lambda$ in Eq. (3.41) is proportional to the lateral system size $\lambda \sim A^{-1 /(d-1)}$ because $P$ stems from a lateral momentum [see, e.g., Eq. (3.37)]. In $d=3$ dimensions Eq. (3.41) leads to

$$
p \stackrel{d=3}{\simeq} t^{-3 / 4} \frac{\Gamma(3 / 4) K_{d-1}}{8 \pi^{2} A L} \ln \left(\frac{\sigma}{\lambda t^{1 / 4}}\right) .
$$

Once this problematic contribution has been removed from Eq. (3.38), the continuum approximation leading to Eq. (3.40) can again be applied, such that, in total, the short-time scaling behavior is given by the sum of Eqs. (3.40) and (3.41). Specifically in $d=3$, this renders

$$
\left.\left\langle\Delta \mathcal{P}_{f}^{(\mathrm{p})}\left(t \ll \min \left(L^{4}, \tau^{-2}\right)\right) \Delta \mathcal{P}_{f}^{(\mathrm{p})}(0)\right)\right|_{d=3} \simeq t^{-1} \frac{H_{d}}{A}+t^{-3 / 4} \frac{\Gamma(3 / 4)}{8 \pi^{2} A L} \ln \frac{\sigma}{\lambda t^{1 / 4}},
$$

where the quantity $H_{d}$ is defined in Eq. (3.34).

Case $t \gg \tau^{-2}$. From $t \gg \tau^{-2}$, it follows that $t^{-1 / 4} \gg(\tau t)^{-1 / 2}$. The form of the exponential terms in Eq. (3.38) then implies that $(\tau t)^{-1 / 2}$ provides an upper bound for $q$ below which the integrand contributes. Thus, in this long-time regime, one has 
$q^{2} / \tau \lesssim 1 /\left(t^{1 / 2} \tau\right) \lesssim 1,{ }^{9}$ such that the square brackets in Eq. (3.38) essentially reduce to $\tau^{2}$. We apply this approximation analogously to the exponentials in Eq. (3.38) and distinguish the two cases $\tau t \gg L^{2}$ and $\tau t \ll L^{2}$. In the first case we have $\tau^{2} t \gg \max \left(1, \tau L^{2}\right)$, which allows us to set $k_{m}=k_{n}=0$. Under these assumptions, Eq. (3.38) renders the following long-time behavior of the film pressure correlations:

$$
\left\langle\Delta \mathcal{P}_{f}^{(\mathrm{p})}\left(t \gg L^{2} / \tau\right) \Delta \mathcal{P}_{f}^{(\mathrm{p})}(0)\right\rangle \simeq \frac{1}{2^{(3 d-1) / 2} \pi^{(d-1) / 2} A L^{2}}(\tau t)^{-(d-1) / 2}
$$

For $\tau^{-1} \ll \tau t \ll L^{2}$, on the other hand, we can evaluate Eq. (3.38) by replacing the sum over $k_{j}$ by an integral. As before, this is equivalent to inserting Eq. (2.25) into Eq. (3.14), keeping only the dominant term $(1 / 2)\left(\tau \mathcal{C}^{(\mathrm{p})}\right)^{2}$. This gives the following intermediate asymptotic behavior:

$$
\left\langle\Delta \mathcal{P}_{f}^{(\mathrm{p})}\left(\tau^{-2} \ll t \ll L^{2} / \tau\right) \Delta \mathcal{P}_{f}^{(\mathrm{p})}(0)\right\rangle \simeq \frac{1}{2^{3(d+1) / 2} \pi^{(d+1) / 2} A}(\tau t)^{-(d+1) / 2} .
$$

Upon increasing $L$, the long-time behavior in Eq. (3.44) is gradually shifted towards later times and is replaced by the behavior in Eq. (3.45), which coincides with the long-time behavior of the bulk pressure reported in Eq. (3.36). Analogously to Eq. (3.39), the asymptotic long-time behavior in Eq. (3.44) is a specific consequence of the continuous spectrum of zero modes in the transverse direction of a thin film.

\section{Neumann BCs}

According to Eq. (3.16), the dynamic correlations of the film pressure for Neumann BCs can be fully expressed in terms of the dynamic OP correlation function $\mathcal{C}^{(\mathrm{p})}$ [Eq. (2.27)] for periodic BCs. Inserting Eq. (2.27) into Eq. (3.16), we obtain

$$
\begin{aligned}
\left\langle\Delta \mathcal{P}_{f}^{(\mathrm{N})}(t) \Delta \mathcal{P}_{f}^{(\mathrm{N})}(0)\right\rangle & =\left\langle\mathcal{P}_{f}^{(\mathrm{N})}(t) \mathcal{P}_{f}^{(\mathrm{N})}(0)\right\rangle-\left\langle\mathcal{P}_{f}^{(\mathrm{N})}\right\rangle^{2} \\
& =\frac{K_{d-1}}{2 A L^{2}} \sum_{m, n=-\infty}^{\infty} \int_{0}^{\infty} d p S\left(p, k_{m}, t\right) S\left(p, k_{n}, t\right) p^{d-2}\left[2\left(k_{n}^{2}+p^{2}\right)\left(k_{n}^{2}+k_{m}^{2}\right)+p^{4}+2 \tau\left(2 k_{n}^{2}+p^{2}\right)+\tau^{2}\right],
\end{aligned}
$$

where the constant $K_{d}$ and the function $S$ are defined as in Eq. (3.38). We emphasize that, although the sum runs over all integers, the wave numbers $k_{m, n}$ are the ones for Neumann BCs [Eq. (2.31a)]. This is a consequence of the prescription in Eq. (3.16), according to which the wave numbers $k_{n, m}^{(\mathrm{p})}$ [Eq. (2.28)] entering the expressions of $\mathcal{C}^{(\mathrm{p})}$ are to be evaluated with $2 L$ instead of $L$.

As it was the case for periodic BCs, we find that $\left\langle\left(\Delta \mathcal{P}_{f}^{(\mathrm{N})}\right)^{2}(t)\right\rangle$ has a weak logarithmic divergence for $d=3$, but is finite for $d>3$ and $t>0$. Proceeding analogously to the previous subsection, one obtains the following asymptotic time behaviors:

$$
\begin{aligned}
&\left\langle\Delta \mathcal{P}_{f}^{(\mathrm{N})}\left(L^{4} \ll t \ll \tau^{-2}\right) \Delta \mathcal{P}_{f}^{(\mathrm{N})}(0)\right) \simeq \frac{1}{2^{(7 d-3) / 4} \pi^{(d-1) / 2} \Gamma(d / 4+1 / 4) A L^{2}} t^{-(d-1) / 4}, \\
&\left\langle\Delta \mathcal{P}_{f}^{(\mathrm{N})}\left(t \gg \max \left(\tau^{-2}, L^{2} / \tau\right)\right) \Delta \mathcal{P}_{f}^{(\mathrm{N})}(0)\right) \simeq \frac{1}{2^{(3 d-1) / 2} \pi^{(d-1) / 2} A L^{2}}(\tau t)^{-(d-1) / 2}, \\
&\left\langle\Delta \mathcal{P}_{f}^{(\mathrm{N})}\left(\tau^{-2} \ll t \ll L^{2} / \tau\right) \Delta \mathcal{P}_{f}^{(\mathrm{N})}(0)\right\rangle \simeq \frac{1}{2^{(3 d-1) / 2} \pi^{(d+1) / 2} A}(\tau t)^{-(d+1) / 2}, \\
&\left.\left\langle\Delta \mathcal{P}_{f}^{(\mathrm{N})}\left(t \ll \min \left(L^{4}, \tau^{-2}\right)\right) \Delta \mathcal{P}_{f}^{(\mathrm{N})}(0)\right)\right|_{d \geqslant 4} \simeq t^{-(1+d) / 4} \frac{H_{d}^{(\mathrm{N})}}{A}, \quad H_{d}^{(\mathrm{N})} \equiv \Omega_{d-1} \int_{0}^{\infty} d \psi F_{d}^{(\mathrm{N})}(\psi), \\
&\left.\left\langle\Delta \mathcal{P}_{f}^{(\mathrm{N})}\left(t \ll \min \left(L^{4}, \tau^{-2}\right)\right) \Delta \mathcal{P}_{f}^{(\mathrm{N})}(0)\right\rangle\right|_{d=3} \simeq t^{-1} \frac{H_{d}^{(\mathrm{N})}}{A}+t^{-3 / 4} \frac{\Gamma(3 / 4)}{4 \pi^{2} A L} \ln \frac{\sigma}{\lambda t^{1 / 4}},
\end{aligned}
$$

where the function $F_{d}^{(\mathrm{N})}(\psi)$ follows from Eq. (3.16) by replacing the film correlator $\mathcal{C}^{(\mathrm{p})}$ by the bulk correlator $\mathcal{C}_{b}$ [Eq. (2.26)]. $F_{d}^{(\mathrm{N})}$ is given by a rather lengthy expression and is not stated here. The meaning of the parameters $\lambda$ and $\sigma$ is the same as in Eq. (3.43). The quantities $H_{d}^{(\mathrm{N})}$ are finite for $d>2$ and their numerical values are $H_{3}^{(\mathrm{N})}=2.005 \times 10^{-2}$ and $H_{4}^{(\mathrm{N})}=2.250 \times$ $10^{-3}$. We note that $H_{d}^{(\mathrm{N})} \approx 4 H_{d}^{(\mathrm{p})}$ holds only approximately because the expression in Eq. (3.16) is not a multiple of the one in Eq. (3.14). Remarkably, this difference, i.e., $H_{n}^{(\mathrm{N})} \neq 4 H_{d}^{(\mathrm{p})}$, does not affect most of the other asymptotic scaling laws in Eq. (3.47); in fact, the behaviors at long times [Eqs. (3.47a) and (3.47b)] coincide with the ones [Eqs. (3.39) and (3.44)] pertaining to periodic BCs. An exception is the intermediate asymptotic behavior in Eq. (3.47c), which equals four times the one in Eq. (3.45).

\footnotetext{
${ }^{9}$ This relation follows by using $\tau t^{1 / 2} \gtrsim 1$ in the inequality $q^{2} / \tau \lesssim 1 /\left(\tau^{2} t\right)$.
} 


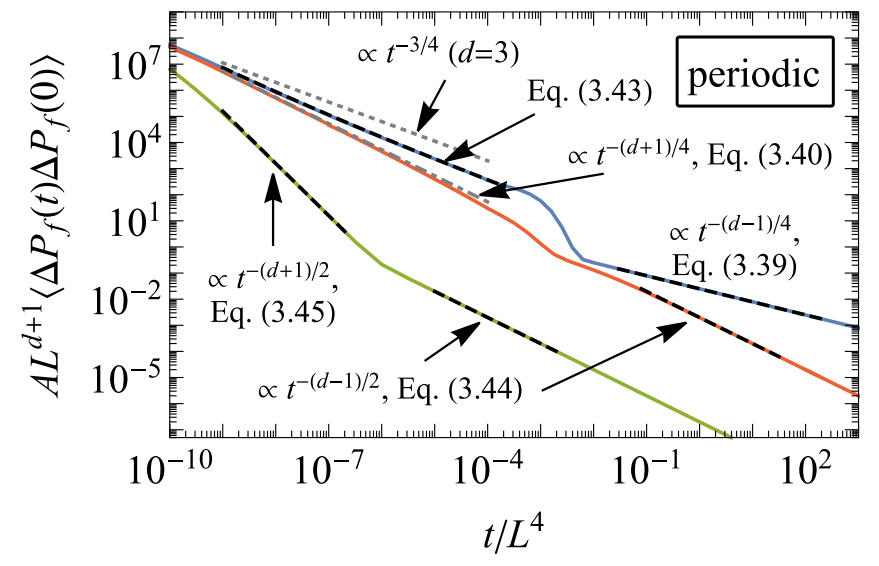

(a)

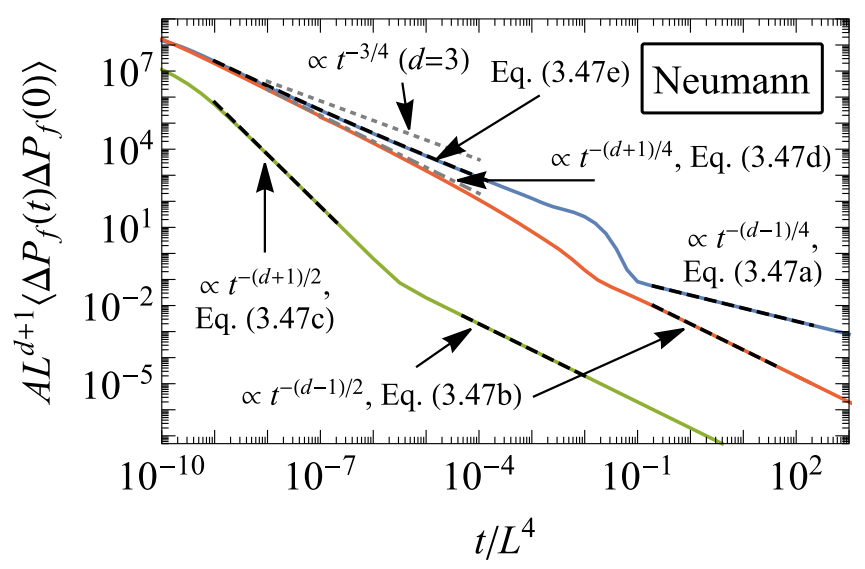

(b)

FIG. 2. Dynamic correlations $\left\langle\Delta \mathcal{P}_{f}(t) \Delta \mathcal{P}_{f}(0)\right\rangle$ of the film pressure for (a) periodic BCs [Eq. (3.38)] and (b) Neumann BCs [Eq. (3.46)] for $d=3$ and various temperatures [ $L^{2} \tau=0$ (blue), 10 (red), $10^{5}$ (green), from top to bottom]. The solid curves are obtained by numerically evaluating Eqs. (3.38) and (3.46), while the dashed lines represent the asymptotic laws reported in Eqs. (3.39), (3.40), and (3.43)-(3.45) for (a) and in Eq. (3.47) for (b). The gray dotted line corresponds to a decay $\propto t^{-3 / 4}$ (applying to $d=3$ ), while the gray dashed-dotted line represents Eqs. (3.40) and (3.47d). These lines are drawn in order to highlight the influence of the logarithmic correction for $d=3$ [see Eqs. (3.42) and (3.47e)]. For the evaluation of $\left\langle\Delta \mathcal{P}_{f}(t) \Delta \mathcal{P}_{f}(0)\right\rangle$ at bulk criticality $(\tau=0)$ we used a low momentum cutoff $\lambda \simeq 10^{-9} / L$ (this specific value is chosen for illustrative purposes). For times shorter than those covered by the plot, the curves corresponding to $L^{2} \tau=10^{5}$ (green) and 10 (red) approach the asymptotic critical ( $\tau=0$ ) short-time behavior given in Eq. (3.43). Due to the smaller value of $L^{2} \tau$, the red curve lies closer to this asymptote and crosses over to the off-critical asymptote [Eqs. (3.44) and (3.47b)] later than the green one. Except for the case of the power law $\propto t^{-3 / 4}$, the asymptotic laws reported in these plots do not involve a fitting parameter. We note that $\left\langle\Delta \mathcal{P}_{f}(t) \Delta \mathcal{P}_{f}(0)\right\rangle$ has the same dimension as $A^{-1} L^{-(d+1)}$ [see the comment after Eq. (3.10)].

\section{Discussion}

By comparing Eqs. (3.36), (3.39), and (3.47a) we infer that, at sufficiently long times, only the contribution of the film pressure is relevant for the correlation function of the CCF [Eq. (3.33)]. Conversely, at short times, the correlation functions of the film and the bulk pressure are both of similar magnitude [see Eqs. (3.34), (3.40), and (3.47d)].

Figure 2 summarizes the behavior of the film pressure $\left\langle\Delta \mathcal{P}_{f}(t) \Delta \mathcal{P}_{f}(0)\right\rangle$ for (a) periodic and (b) Neumann BCs as a function of time. The solid curves in Fig. 2 are based on a numerical evaluation of Eqs. (3.38) and (3.46), respectively, while the broken lines indicate the various asymptotic power laws obtained in the preceding subsections. Note that these asymptotic predictions do not involve a fitting parameter. In order to regularize the logarithmic infrared divergence of $\left\langle\Delta \mathcal{P}_{f}(t) \Delta \mathcal{P}_{f}(0)\right\rangle$ in $d=3$ at bulk criticality $(\tau=0)$ [see Eqs. (3.43) and (3.47e)], we use a value $\lambda=10^{-9} / L$ for the infrared cutoff. The value of the prefactor is sufficiently small in order to satisfy the condition of considering a thin film, but otherwise arbitrary and chosen for illustrative purposes. For the parameter $\sigma$, which is rather technical and the origin of which is explained in the context of Eq. (3.41), we use a value $\sigma \simeq 0.1 / L$ motivated by numerical considerations. We remark that, due to the logarithmic dependence, using values of these parameters which differ even by an order of magnitude does not noticeably affect the quality of the asymptotic approximations.

\section{FLUCTUATIONS OF THE BOUNDARY}

In the preceding section, we have studied static and dynamic pressure correlations at a spatially fixed boundary of the film. We now relax this assumption and consider a responsive, i.e., movable film boundary.

\section{A. Dynamical model}

We start with a description in terms of the physical time $\tilde{t}$ [see Eq. (2.9)] and denote the position of the film boundary by $R(\tilde{t})$, which initially is at $R_{0} \equiv R(\tilde{t}=0)=L$ (see Fig. 1$)$. We consider the motion of the boundary to be overdamped and subject to the instantaneous force $\mathcal{K}_{z}$ [Eq. (2.16)] as well as to Gaussian white noise $\eta$ with covariance $\left\langle\eta(\tilde{t}) \eta\left(\tilde{t}^{\prime}\right)\right\rangle=$ $2 \delta\left(\tilde{t}-\tilde{t}^{\prime}\right)$. Accordingly, we propose the following Langevin equation for the time evolution of $R$ :

$$
\begin{aligned}
\partial_{\tilde{t}} R(\tilde{t}) & =\Gamma T A \mathcal{K}(R(\tilde{t}), \tilde{t})+\sqrt{\Gamma T} \eta(\tilde{t}) \\
& =\Gamma T A\langle\mathcal{K}(R(\tilde{t}))\rangle_{\phi}+\Gamma T A \Delta \mathcal{K}(R(\tilde{t}), \tilde{t})+\sqrt{\Gamma T} \eta(\tilde{t}),
\end{aligned}
$$

where $\Gamma$ is a mobility coefficient [which has the same dimension as $\left.L^{2} /(T \tilde{t})\right] .{ }^{10}$ The prefactor $T A$ in front of $\mathcal{K}$ arises because we have defined $\mathcal{K}$ as a force per area $A$ of the film at temperature $T$ [see Eq. (2.18)]. A reflecting boundary, representing a wall impenetrable to the moving boundary, is taken to be positioned at $R=0$. In the second equation in Eq. (4.1),

\footnotetext{
${ }^{10}$ The mobility $\Gamma$ is expected to be proportional to the mobility $\gamma$ of the OP field [Eq. (2.9)] [41,42].
} 
the CCF $\mathcal{K}$ is split according to Eq. (3.3) into a mean $\langle\mathcal{K}\rangle_{\phi}$ and a fluctuating part $\Delta \mathcal{K}$ (with respect to the OP field). Since we consider thermal equilibrium for the OP field, the mean force $\langle\mathcal{K}\rangle$ does not explicitly depend on time. The white noise $\eta$ accounts for the molecular momentum exchange between the solvent and the surface, which would be present even without a coupling to the OP. Upon introducing the rescaled time $t=\gamma \tilde{t}$ as defined in Eq. (2.11), Eq. (4.1) turns into

$$
\begin{aligned}
\partial_{t} R(t) & =D A\langle\mathcal{K}(R(t))\rangle_{\phi}+D A \Delta \mathcal{K}(R(t), t)+\sqrt{D} \eta(t), \\
D & \equiv T \Gamma / \gamma,
\end{aligned}
$$

where the parameter $D$ is the bare diffusivity with dimension $[D]=\left[L^{-2}\right]$.

The fact that the stochastic process $\Delta \mathcal{K}(R, \tilde{t}))$ has a nonlinear dependence on $R$ raises the issue of the proper stochastic calculus (e.g., Ito or Stratonovich) to be used in Eq. (4.1) [30,43]. In principle, this can be addressed by deriving Eq. (4.1) from a more basic model, e.g., by adiabatically eliminating the OP degrees of freedom from a coupled system of Markovian Langevin equations for the boundary and the fluid medium [24-26,30,44-46]. While this is beyond the scope of this study, it turns out that, in the Markovian limit, the variance of $\Delta \mathcal{K}$ is approximately independent of $R$, rendering the choice of the stochastic calculus to be immaterial for the present analysis (see Sec. IV C 2).

Equation (4.2) describes the random motion of $R$ in the Casimir potential associated with $\langle\mathcal{K}\rangle_{\phi}$, subject to the Brownian noise $\eta$ [see Eq. (4.6) below] and an additional nonMarkovian noise $\Delta \mathcal{K}$. While Eq. (4.2) resembles previously proposed Langevin equations for inclusions in critical media [24-26,47], here we assume that the presence of a movable boundary does not modify the equation of motion of the OP [Eq. (2.12)]. In fact, the only effect of the boundary on the $\mathrm{OP}$ is to impose BCs [see Eq. (2.8c)]. Accordingly, the noise provided by $\Delta \mathcal{K}$ is not balanced by a corresponding friction term, implying that detailed balance is not satisfied by the model defined by Eqs. (4.2) and (2.12). This is also reflected by the fact that the distribution of the boundary position does not approach the expected equilibrium form at long times (see Appendix). We thus refer to our model as having a "passive boundary." For a more adequate description of the dynamics of a colloidal particle in a critical fluid in thermal equilibrium $[5,8,23,28]$, detailed balance has to be reestablished. ${ }^{11}$ This can be accounted for by either adding appropriate coupling terms to the OP dynamics in Eq. (2.12) (see Ref. [47]), or by suitably modifying Eq. (4.2) in order to satisfy the fluctuationdissipation theorem. We will return to the latter approach in Sec. IV C 2 below. However, compared with the models in Refs. [24-26,47], a distinctive feature of the present model is that it allows one to enforce strict no-flux BCs at the location of a boundary.

\footnotetext{
${ }^{11}$ In addition to the limitations of the model spelled out here, experimentally, the surface of the colloid and the wall typically exhibit strong adsorption of the OP. A refined theory would thus not only have to account for the nonplanar surface of the colloid, but also consider the so-called $(+ \pm)$ BCs [23] instead of periodic or Neumann ones.
}

The mean equilibrium CCF (per area $A$ and temperature $T$ ) has the form

$$
\langle\mathcal{K}(R)\rangle_{\phi}=R^{-d} \Xi(R / \xi),
$$

where $\Xi$ is a universal scaling function depending on the boundary conditions. Explicit expressions within the Gaussian approximation are reported in, e.g., Refs. $[40,48]$. At criticality (i.e., $\xi \rightarrow \infty$ ), one has $\Xi(0)<0$ for periodic and Neumann BCs [see Eq. (3.32)]. At short distances, the electrostatic repulsion between the fixed wall and the mobile boundary must be taken into account [23,49], thereby regularizing also the singularity of the CCF [Eq. (4.3)] appearing within the continuum description in the limit $R \rightarrow 0$. Phenomenologically, this electrostatic repulsion can be described by the potential (defined, analogously to the CCF, per area $A$ and temperature $T$ ) [5]

$$
V_{\mathrm{es}}(z)=\alpha^{-(d-1)} e^{-z / \ell_{D}},
$$

where $\alpha$ is an effective parameter (of the same dimension as $L$ ) and $\ell_{D}$ denotes the Debye screening length. Introducing formally the Casimir potential $V_{\mathrm{C}}(z)$ associated with the mean CCF via $\langle\mathcal{K}(z)\rangle_{\phi}=-d V_{\mathrm{C}}(z) / d z$, we define an effective potential

$$
\mathcal{U}(z)=V_{\mathrm{es}}(z)+V_{\mathrm{C}}(z),
$$

which gives rise to a force $-\mathcal{U}^{\prime}(z)$ replacing $\langle\mathcal{K}(z)\rangle_{\phi}$ in Eq. (4.2). Accordingly, Eq. (4.2) is replaced by the Langevin equation

$$
\partial_{t} R(t)=-D A \mathcal{U}^{\prime}(R(t))+D A \Delta \mathcal{K}(R(t), t)+\sqrt{D} \eta(t) .
$$

The effective potential $\mathcal{U}(z)$ has a minimum at a finite distance $z_{0}$ from the wall. In order to facilitate an analytical treatment, we consider its quadratic approximation around its minimum:

$$
\mathcal{U}(z) \simeq \frac{1}{2} \kappa\left(z-z_{0}\right)^{2} .
$$

An explicit expression for the (temperature-dependent) parameter $\kappa$ (which has the same dimension as $L^{-d-1}$ ) can in principle be obtained from Eqs. (4.3) and (4.4), but is not necessary in the following. As a further simplification, we focus on the limit in which the dynamics of the field $\phi$ is faster than that of the position $R$ (adiabatic approximation). Since within the Gaussian model B [35] the relaxation time of a critical OP fluctuation grows $\propto \mathcal{L}^{4}$, where $\mathcal{L}$ is the characteristic system size, the adiabatic approximation requires that both the film and the "bulk" part of the system are finite (see Fig. 1). Still, we take the bulk to be sufficiently large so that a continuum approximation is appropriate. Requiring $\mathcal{L}$ to be a measure of the largest length scale in the system (which implies also $\mathcal{L} \gtrsim A^{1 /(d-1)}$ ) allows us to introduce a dimensionless "adiabaticity" parameter

$$
\chi \equiv D \mathcal{L}^{2} .
$$

In terms of $\chi$, Eq. (4.2) can be expressed as

$$
\partial_{t} R(t)=-\chi \frac{A \mathcal{U}^{\prime}(R(t))}{\mathcal{L}^{2}}+\chi \frac{A \Delta \mathcal{K}(R(t), t)}{\mathcal{L}^{2}}+\sqrt{\chi} \frac{\eta(t)}{\mathcal{L}} .
$$

Thus, the adiabatic limit requires $\chi \ll 1$ and facilitates a perturbative solution of Eq. (4.9). For typical experimental 
conditions of a colloid immersed in a critical solvent, the condition $\chi \ll 1$ is fulfilled [28].

\section{B. Boundary located in the bulk}

Here, we assume here that $R(t)$ is sufficiently far from the wall such that the effective force $\mathcal{U}^{\prime}$ in Eq. (4.6) can be neglected. Accordingly, we have a bulklike system on both sides of the boundary. Integrating Eq. (4.6) in time renders

$$
R(t)-R(0)=D A \int_{0}^{t} d s \Delta \mathcal{K}(R(s), s)+B(t)
$$

where $B(t) \equiv \sqrt{D} \int_{0}^{t} d s \eta(s)$ is a Brownian motion with

$$
\langle B(t)\rangle=0 \quad \text { and } \quad\left\langle B(t) B\left(t^{\prime}\right)\right\rangle=2 D \min \left(t, t^{\prime}\right) .
$$

Upon squaring both sides of Eq. (4.10) and averaging over the distribution of the position $R$ and the equilibrium configurations of the field $\phi$ [denoted by $\langle\ldots\rangle=\left\langle\langle\ldots\rangle_{\phi}\right\rangle_{R}$, where $\langle\ldots\rangle_{\phi} \equiv \int \mathcal{D} \phi \ldots P_{\text {eq }}$ with $P_{\text {eq }}$ reported in Eq. (2.1)], one finds the mean-squared displacement (MSD)

$$
\left\langle[R(t)-R(0)]^{2}\right\rangle=2 D t+2 D^{2} A \mathcal{M}_{b}(t)
$$

with

$$
\mathcal{M}_{b}(t) \equiv \frac{A}{2} \int_{0}^{t} d s \int_{0}^{t} d s^{\prime}\left\langle\Delta \mathcal{K}(R(s), s) \Delta \mathcal{K}\left(R\left(s^{\prime}\right), s^{\prime}\right)\right\rangle,
$$

while the cross term $\int_{0}^{t} d s\left\langle\langle\Delta \mathcal{K}(R(s), s)\rangle_{\phi} B(t)\right\rangle_{R}$ vanishes because $\langle\Delta \mathcal{K}\rangle=0$ [see Eq. (3.3)]. The quantity $\mathcal{M}_{b}$ represents the fluctuation contribution to the MSD, which, according to Eq. (3.4), is determined in the present case by the bulk pressure correlations:

$$
\mathcal{M}_{b}(t)=A \int_{0}^{t} d s \int_{0}^{t} d s^{\prime}\left\langle\Delta \mathcal{P}_{b}(R(s), s) \Delta \mathcal{P}_{b}\left(R\left(s^{\prime}\right), s^{\prime}\right)\right\rangle .
$$

The factor 2 in Eq. (4.12) arises because the bulk pressure acts on both sides of the boundary, while in Eq. (4.13) the prefactor $A$ is introduced in order to render $\mathcal{M}$ independent of $A$. We note that, due to the presence of $\Delta \mathcal{K}$, the process described by Eq. (4.2) is highly nonlinear.

In order to evaluate Eq. (4.13), we note that in Eq. (4.9) the OP-induced noise $\Delta \mathcal{K}$ is of higher order in $\chi$ than the white noise $\eta$. Accordingly, solving Eq. (4.9) (assuming, as before, $\mathcal{U}^{\prime}=0$ ) in the regime $\chi \ll 1$ to first order in $\chi$ yields a free Brownian motion for $R(t)$ :

$$
R(t) \simeq R(0)+B(t)
$$

which is to be used in Eq. (4.14) (see Sec. IV D below). The required two-time joint probability distribution for a free Brownian process is given by [30]

$$
\begin{aligned}
p\left(R_{s}, R_{s^{\prime}}, R_{0}, s, s^{\prime}\right)= & \frac{1}{4 \pi D \sqrt{s s^{\prime}-\min \left(s, s^{\prime}\right)}} \\
& \times \exp \left\{-\frac{1}{4 D\left[s s^{\prime}-\min \left(s, s^{\prime}\right)\right]}\left[s^{\prime}\left(R_{s}-R_{0}\right)^{2}+s\left(R_{s^{\prime}}-R_{0}\right)^{2}-2 \min \left(s, s^{\prime}\right)\left(R_{s}-R_{0}\right)\left(R_{s^{\prime}}-R_{0}\right)\right]\right\}
\end{aligned}
$$

which renders the following variants of the characteristic function:

$$
\begin{aligned}
\left\langle e^{i Q\left(R(s)-R\left(s^{\prime}\right)\right)}\right\rangle & =\int_{-\infty}^{\infty} d R_{1} \int_{-\infty}^{\infty} d R_{2} p\left(R_{1}, R_{2}, R_{0}, s, s^{\prime}\right) e^{i Q\left(R_{1}-R_{2}\right)}=e^{-D Q^{2}\left|s-s^{\prime}\right|}, \\
\left\langle e^{i Q\left(R(s)+R\left(s^{\prime}\right)\right)}\right\rangle & =e^{2 i Q R_{0}-D Q^{2}\left[s+s^{\prime}+2 \min \left(s, s^{\prime}\right)\right]} .
\end{aligned}
$$

\section{Boundary located near a wall}

Here, we retain the effective force $\mathcal{U}^{\prime}$ in Eqs. (4.6) and (4.9). Within the regime $\chi \ll 1$, the fluctuating contribution of the CCF $\Delta \mathcal{K}$ is subdominant compared to the white noise $\eta$, while the deterministic term related to $\mathcal{U}^{\prime}$ has to be regarded as formally to be of the same order in $\chi$ as $\eta$. (This assignment is also applied, e.g., upon solving a standard Langevin equation of a Brownian particle [30].) Accordingly, for $\chi \ll 1$, the leading order solution $R(t)$ of Eq. (4.9) [based on the assumption formulated in Eq. (4.7)] is an Ornstein-Uhlenbeck process [30]. However, the calculation of an average as in Eq. (4.13) over this process leads to intractable expressions. We therefore solve Eq. (4.9) instead by applying two complementary approaches: in one (see Sec. IV C 1), we formally integrate Eq. (4.9) in time and focus on short times, in which case the Ornstein-Uhlenbeck process reduces to a free Brownian motion. In a second approach (see Sec. IV C 2), we determine a Markovian approximation of $\Delta \mathcal{K}$ in the adiabatic limit $\chi \ll 1$. Although the second approach holds at all times, we use it specifically to study the long-time behavior.

\section{Direct integration of Eq. (4.9)}

Upon integrating Eq. (4.9) [or, equivalently, Eq. (4.6)] in time one obtains the formal solution

$R(t)-R(0)=D A \int_{0}^{t} d s\left[-\mathcal{U}^{\prime}(R(s))+\Delta \mathcal{K}(R(s), s)\right]+B(t)$,

with the Brownian motion $B(t)$ [Eq. (4.11)]. Upon squaring both sides of Eq. (4.18) and averaging over the (yet unknown) distribution of the boundary position and of the OP field, we 
obtain

$$
\begin{aligned}
\left\langle[R(t)-R(0)]^{2}\right\rangle= & 2 D t-D A \int_{0}^{t} d s\left\langle\mathcal{U}^{\prime}(R(s)) B(t)\right\rangle_{R} \\
& +(D A)^{2} \int_{0}^{t} d s \int_{0}^{t} d s^{\prime}\left[\left\langle\mathcal{U}^{\prime}(R(s)) \mathcal{U}^{\prime}\left(R\left(s^{\prime}\right)\right)\right\rangle\right. \\
& \left.+\left\langle\Delta \mathcal{K}(R(s), s) \Delta \mathcal{K}\left(R\left(s^{\prime}\right), s^{\prime}\right)\right\rangle\right],
\end{aligned}
$$

while, within the approximation eventually considered in Eq. (4.22) below, the cross term $\quad \int_{0}^{t} d s\left[(D A)^{2}\left\langle\mathcal{U}^{\prime}(R(s))\langle\Delta \mathcal{K}(R(s), s)\rangle_{\phi}\right\rangle_{R}+\right.$ $D A\left\langle\langle\Delta \mathcal{K}(R(s), s)\rangle_{\phi} B(t)\right\rangle_{R}$ ] vanishes since $\langle\Delta \mathcal{K}\rangle=0$ [see Eq. (3.3)] and $R(s)$ is unaffected by $\phi$. The fluctuating contribution to the MSD is represented by the last term in Eq. (4.19), which, according to Eq. (3.4), consists of the correlations of the film and the bulk pressure:

$$
\begin{aligned}
& A \int_{0}^{t} d s \int_{0}^{t} d s^{\prime}\left\langle\Delta \mathcal{K}(R(s), s) \Delta \mathcal{K}\left(R\left(s^{\prime}\right), s^{\prime}\right)\right\rangle \\
& \quad=\mathcal{M}_{f}(t)+\mathcal{M}_{b}(t)
\end{aligned}
$$

where $\mathcal{M}_{b}$ is given by Eq. (4.14) and

$$
\mathcal{M}_{f}(t) \equiv A \int_{0}^{t} d s \int_{0}^{t} d s^{\prime}\left\langle\Delta \mathcal{P}_{f}(R(s), s) \Delta \mathcal{P}_{f}\left(R\left(s^{\prime}\right), s^{\prime}\right)\right\rangle .
$$

In the limit $\chi \ll 1$, the leading order solution of Eq. (4.9) is an Ornstein-Uhlenbeck process, which, at short times $t \ll$ $1 /(D A \kappa)$, reduces to a free Brownian motion [see Eq. (4.11)]:

$$
R(t) \simeq R(0)+B(t), \quad R(0)=z_{0} \quad[t \ll 1 /(D A \kappa)] .
$$

Note that, in order to to simplify the calculation, we consider the film boundary to be initially located at the potential minimum $z_{0}$. Upon using Eqs. (4.16), (4.17), and (4.22), the integrals involving $\mathcal{U}^{\prime}(z)=\kappa\left(z-z_{0}\right)$ in Eq. (4.19) can be readily evaluated, yielding in the short-time limit

$$
\left\langle[R(t)-R(0)]^{2}\right\rangle \stackrel{t \ll 1 /(D A \kappa)}{\simeq} 2 D t+D^{2} A\left[\mathcal{M}_{f}(t)+\mathcal{M}_{b}(t)\right]+O\left(\chi^{3}\right) .
$$

\section{Markovian approximation of $\Delta \mathcal{K}$}

Here, we seek an alternative solution of Eq. (4.9) which is valid for all times. In order to identify a suitable approximation, we define a new time variable $\hat{t}$ via

$$
t=\hat{t} / \chi
$$

and introduce the quantities $\hat{R}(\hat{t})=R(\hat{t} / \chi), \quad \hat{\eta}(\hat{t})=$ $\frac{1}{\sqrt{\chi}} \eta(\hat{t} / \chi)\left[\right.$ with $\left\langle\hat{\eta}(\hat{t}) \hat{\eta}\left(\hat{t}^{\prime}\right)=2 \delta\left(\hat{t}-\hat{t}^{\prime}\right)\right]$. In terms of them, Eq. (4.9) becomes

$$
\partial_{\hat{t}} \hat{R}(\hat{t})=\frac{A\langle\mathcal{K}(\hat{R}(\hat{t}))\rangle_{\phi}}{\mathcal{L}^{2}}+\frac{A \Delta \mathcal{K}(\hat{R}(\hat{t}), \hat{t} / \chi)}{\mathcal{L}^{2}}+\frac{\hat{\eta}(\hat{t})}{\mathcal{L}},
$$

where, using the time-translation invariance of the CCF fluctuations, the correlation function of the field-induced noise $\Delta \mathcal{K}$ [see Eqs. (3.4) and (3.10)] is given by

$$
\hat{\mathcal{N}}\left(\hat{R}, \hat{R}^{\prime}, \hat{s}-\hat{s}^{\prime}\right) \equiv\left\langle\Delta \mathcal{K}(\hat{R}, 0) \Delta \mathcal{K}\left(\hat{R}^{\prime},\left(\hat{s}-\hat{s}^{\prime}\right) / \chi\right)\right\rangle .
$$

In the limit $\chi \ll 1$, one expects from Eqs. (4.25) and (4.26) that $\Delta \mathcal{K}$ decorrelates rapidly in time, justifying a Markovian approximation for $\hat{\mathcal{N}}$. Furthermore, we assume $\Delta \mathcal{K}$ to be Gaussian, such that cumulants higher than the second one reported in Eq. (4.26) are zero. ${ }^{12}$ From standard adiabatic elimination techniques $[30,50]$ it follows that $\Delta \mathcal{K}$ can be approximated by a Markovian noise $\Delta \mathcal{K}_{M}$ with the variance

$$
\left\langle\Delta \mathcal{K}_{M}(R(t), t) \Delta \mathcal{K}_{M}\left(R\left(t^{\prime}\right), t^{\prime}\right)\right\rangle=2 \mathcal{N}(R(t)) \delta\left(t-t^{\prime}\right), \quad \mathcal{N}(R) \equiv \int_{0}^{\infty} d u \hat{\mathcal{N}}(R, R, u) .
$$

The resulting Stratonovich-type Langevin equation [30,50] is given by

$$
\partial_{t} R(t)=-D A \mathcal{U}^{\prime}(R(t))+D A \Delta \mathcal{K}_{M}(R(t), t)+\sqrt{D} \eta(t)
$$

and corresponds to the following Fokker-Planck equation for the probability density $P(R, t)$ of the position $R$ of the movable boundary:

$$
\partial_{t} P(R, t)=\partial_{R}\left[\left(D A \mathcal{U}^{\prime}(R)-\frac{1}{2}(D A)^{2} \mathcal{N}^{\prime}(R)\right) P(R, t)\right]+\partial_{R}^{2}\left[\left(D+(D A)^{2} \mathcal{N}(R)\right) P(R, t)\right],
$$

where the term involving $\mathcal{N}^{\prime}(R)$ is a consequence of the Stratonovich stochastic calculus, also referred to as "spurious" drift [30]. The expression for the noise amplitude $\mathcal{N}(R)$ (which has the same dimension as $L^{4-2 d}$ ) is obtained by using Eqs. (2.27), (2.32b), (3.4), and (3.10) in Eq. (4.27):

$$
\mathcal{N}(R)=\frac{K_{d-1}}{A} \int_{0}^{\infty} d p\left[\frac{1}{L^{2}} \sum_{m, n=-\infty}^{\infty} \mathcal{Q}^{(\mathrm{p}, \mathrm{N})}\left(k_{m}^{(\mathrm{p}, \mathrm{N})}, k_{n}^{(\mathrm{p}, \mathrm{N})}, p\right)+\int_{-\infty}^{\infty} \frac{d k_{m}}{2 \pi} \int_{-\infty}^{\infty} \frac{d k_{n}}{2 \pi} \mathcal{Q}_{b}\left(k_{m}, k_{n}, p\right)\right],
$$

\footnotetext{
${ }^{12}$ The noise $\Delta \mathcal{K}$ is, in general, not Gaussian, because it follows by applying the nonlinear transformations, given by Eqs. (2.18), (2.19), (2.20), and (3.3), to the (Gaussian) field $\phi$ defined by the stochastic process in Eq. (2.12). Accordingly, the assumption of $\Delta \mathcal{K}$ being Gaussian is necessarily an approximation. We recall that, at least in the static case, Monte Carlo simulations have confirmed the Gaussian character of $\Delta \mathcal{K}[17]$.
} 
where the wave numbers used in the sum are defined in Eq. (2.28) [see also the comment after Eq. (3.46)]; $K_{d}$ is defined after Eq. (3.38), and

$$
\begin{aligned}
\mathcal{Q}_{b}=\mathcal{Q}^{(\mathrm{p})} \equiv & p^{d-2} \frac{3 k_{m}^{2} k_{n}^{2}+2 k_{n}^{4}+2\left(k_{m}^{2}+k_{n}^{2}\right) p^{2}+p^{4}}{\left(k_{m}^{2}+p^{2}\right)\left(k_{n}^{2}+p^{2}\right)\left[k_{m}^{4}+k_{n}^{4}+2\left(k_{n}^{2}+k_{m}^{2}\right) p^{2}+2 p^{4}\right]} \\
\mathcal{Q}^{(\mathrm{N})} \equiv & p^{d-2} \frac{1}{8\left(k_{m}^{2}+p^{2}\right)\left(k_{n}^{2}+p^{2}\right)\left[2 p^{2}\left(k_{m}^{2}+k_{n}^{2}\right)+k_{m}^{4}+k_{n}^{4}+2 p^{4}\right]} \\
& \times\left\{k_{m}^{2}\left[2 p^{2}\left(1+e^{2 i R k_{m}}\right)\left(1+e^{2 i R k_{n}}\right)+k_{n}^{2}\left(3 e^{2 i R\left(k_{m}+k_{n}\right)}+e^{2 i R k_{m}}+e^{2 i R k_{n}}+3\right)\right]\right. \\
& \left.-2 k_{m} k_{n}\left(2 k_{n}^{2}+p^{2}\right)\left(1+e^{2 i R\left(k_{m}+k_{n}\right)}\right)+\left(1+e^{2 i R k_{m}}\right)\left(2 p^{2} k_{n}^{2}+2 k_{n}^{4}+p^{4}\right)\left(1+e^{2 i R k_{n}}\right)\right\}
\end{aligned}
$$

for $\tau=0 .{ }^{13}$ The expressions of these quantities for $\tau \neq 0$ are rather lengthy and thus they are not stated here. In general, the integral over $p$ diverges both at its lower (0) and upper $(\infty)$ limits and thus has to be regularized, which will be discussed below. Symmetry arguments show that $\sum_{m, n} \mathcal{Q}^{(\mathrm{N})}$ is real valued. Note that $\mathcal{Q}^{(\mathrm{N})}$ is also a function of $R$, while $\mathcal{Q}_{b}$ and $\mathcal{Q}^{(\mathrm{p})}$ are not. However, a numerical analysis reveals that $\mathcal{Q}^{(\mathrm{N})}$ is only weakly depending on $R$ (provided $L \lambda \ll 1$ ), which allows us to make the approximation $\mathcal{Q}^{(\mathrm{N})}(R) \simeq \mathcal{Q}^{(\mathrm{N})}(R=0)$ so that $\mathcal{N}^{(\mathrm{N})}(R) \simeq \mathcal{N}^{(\mathrm{N})}(R=0)$. This renders $\mathcal{N}$ to be independent of $R$ for all BCs considered here. Accordingly, Eq. (4.29) can be approximated by

$$
\begin{aligned}
\partial_{t} P(R, t)= & \partial_{R}\left[D A \mathcal{U}^{\prime}(R) P(R, t)\right] \\
& +\partial_{R}^{2}\left\{\left[D+(D A)^{2} \mathcal{N}\right] P(R, t)\right\} .
\end{aligned}
$$

Equation (4.32) describes a passive boundary at position $R$ moving in the harmonic potential $\mathcal{U}$ [Eq. (4.7)] under the influence of the white noise of variance $2 D$ [see Eq. (4.2)] and a Markovian noise of variance $2(D A)^{2} \mathcal{N}$ [see Eq. (4.30)].

Next, we determine the Fokker-Planck equation (FPE) of a boundary in a system with detailed balance. To this end, we assume that the corresponding FPE takes the same form as in Eq. (4.32), up to a different (but still spatially constant) mobility $\Gamma_{\text {eff }}$, i.e.,

$$
\begin{aligned}
\partial_{t} P(R, t)= & \partial_{R}\left[\Gamma_{\text {eff }} A \mathcal{U}^{\prime}(R) P(R, t)\right] \\
& +\partial_{R}^{2}\left\{\left[D+(D A)^{2} \mathcal{N}\right] P(R, t)\right\} .
\end{aligned}
$$

We now require that the fluctuations of the boundary are described by the equilibrium distribution (see Sec. V)

$$
P_{\text {eq }}(R)=\frac{1}{\mathcal{Z}} e^{-A \mathcal{U}(R)}
$$

In order that the steady-state solution of Eq. (4.33) agrees with $P_{\text {eq }}$, the following relation must hold [30]:

$$
\Gamma_{\text {eff }}=D+(D A)^{2} \mathcal{N} .
$$

This can be regarded as a consequence of the fluctuationdissipation theorem [51]. We shall refer to a boundary

\footnotetext{
${ }^{13}$ We recall that $\mathcal{Q}^{(\mathrm{N})}$ is obtained by using Eq. (2.32b), according to which $\mathcal{C}^{(\mathrm{p})}$ is evaluated as it would be for a film of thickness $2 L$ instead of $L$. As a consequence, the wave numbers in Eq. (4.31b) take the same form as those for Neumann BCs [Eq. (2.31a)], but the sum in Eq. (4.30) runs from $-\infty$ to $\infty$.
}

described by the model in Eqs. (4.33) and (4.35) as "thermalized." Importantly, asymptotically at short times, the behaviors of the MSD resulting from Eqs. (4.32) and (4.33) are identical and independent of the mobility (see Sec. IV D). The derivation of the effective mobility of a boundary from a more rigorous approach (following, e.g., Refs. $[41,42,47]$ ) is left for future studies.

Denoting the second term in the square brackets in Eq. (4.30) by $m_{b}$ (where $b$ stands for bulk), power counting (taking into account the integrals over $k_{m}$ and $k_{n}$ ) implies the asymptotic behaviors

$$
\begin{aligned}
\left.m_{b}(p \rightarrow 0, \infty)\right|_{\tau=0} & \propto p^{d-4}, \\
\left.m_{b}(p \rightarrow 0)\right|_{\tau>0} & \propto p^{d-2}, \\
\left.m_{b}(p \rightarrow \infty)\right|_{\tau>0} & \propto p^{d-4} .
\end{aligned}
$$

The first term in the square brackets in Eq. (4.30), denoted by $m_{f}$ (where $f$ stands for film), is dominated for small $p$ by the contribution for $k_{m}=k_{n}=0$ [see Eqs. (2.28) and (2.31a)] and follows the asymptote

$$
\begin{aligned}
& \left.m_{f}(p \rightarrow 0)\right|_{\tau=0} \propto p^{d-6}, \\
& \left.m_{f}(p \rightarrow 0)\right|_{\tau>0} \propto \tau^{-1} p^{d-4},
\end{aligned}
$$

independently of the BCs. For large $p$, a numerical analysis indicates a behavior as in Eq. (4.37a):

$$
m_{f}(p \rightarrow \infty) \propto p^{d-6}
$$

independently of $\tau$. In order to regularize the integral over $p$ in Eq. (4.30), we replace its lower and upper cutoffs by wave numbers $\lambda$ and $\Lambda$, respectively. Accordingly, within the Markovian approximation in $d=3, \mathcal{N}_{b} \equiv \int_{\lambda}^{\Lambda} d p m_{b}$ diverges logarithmically as $\lambda \rightarrow 0$ or $\Lambda \rightarrow \infty$. In contrast, $\mathcal{N}_{f} \equiv \int_{\lambda}^{\Lambda} d p m_{f}$ is finite for $\Lambda \rightarrow \infty$, while it diverges $\propto \mathcal{L}^{2}$ with the characteristic (lateral) system size $\mathcal{L}$ if $\lambda \simeq 1 / \mathcal{L}$ is taken for the lower integration limit.

\section{Short-time behavior of the MSD}

Based on the expressions derived above, in the following we discuss the evolution of the mean-squared displacement (MSD) of the movable boundary at short times and elucidate how the fluctuations of the CCF affect it.

\section{Preliminaries}

The expressions of $\mathcal{M}_{f}$ [Eq. (4.21)] for periodic BCs and $\mathcal{M}_{b}$ [Eq. (4.14)] for the bulk are structurally similar and 
can thus be analyzed together. $\mathcal{M}_{f}^{(\mathrm{p})}$ is obtained by inserting the Fourier transform of the correlation function given in Eq. (2.27) into Eq. (3.13). In contrast to the calculation of the pressure variance at a fixed position discussed in Sec. III C 2, we now have to retain the dependencies on the $z$ coordinate (as well as on the second time argument $t^{\prime}$, which was previously set to 0 ) and subsequently average over the distribution of the boundary position $z=R$. Moreover, we cannot use the simplifications stemming from Eqs. (2.35) and (3.15). In order to keep the expressions tractable, we focus on the bulk critical point $(\tau=0)$.

We illustrate the calculation of $\mathcal{M}_{f}$ by considering a typical contribution to the integrand in Eq. (4.21) for periodic BCs [compare Eq. (3.37) and see Eq. (2.28)]:

$$
\begin{aligned}
\frac{1}{A} & \int d^{d-1} r\left\langle\left[\partial_{\alpha}^{2} \mathcal{C}^{(\mathrm{p})}\left(\mathbf{r}, R(s)-R\left(s^{\prime}\right), s-s^{\prime}\right)\right]\left[\partial_{\alpha^{\prime}}^{2} \mathcal{C}^{(\mathrm{p})}\left(\mathbf{r}, R(s)-R\left(s^{\prime}\right), s-s^{\prime}\right)\right]\right\rangle \\
& =\frac{1}{A L^{2}} \int d^{d-1} r \sum_{m, n=-\infty}^{\infty} \int \frac{d^{d-1} p}{(2 \pi)^{d-1}} \int \frac{d^{d-1} \tilde{p}}{(2 \pi)^{d-1}} p_{\alpha}^{2} \tilde{p}_{\alpha^{\prime}}^{2}\left\langle e^{i(\mathbf{p}+\tilde{\mathbf{p}}) \cdot \mathbf{r}+i\left[R(s)-R\left(s^{\prime}\right)\right]\left(k_{m}+k_{n}\right)}\right\rangle S\left(p, k_{m}, s-s^{\prime}\right) S\left(\tilde{p}, k_{n}, s-s^{\prime}\right) \\
& =\frac{1}{A L^{2}} \sum_{m, n=-\infty}^{\infty} \int \frac{d^{d-1} p}{(2 \pi)^{d-1}} p_{\alpha}^{2} p_{\alpha^{\prime}}^{2}\left\langle e^{i\left[R(s)-R\left(s^{\prime}\right)\right]\left(k_{m}+k_{n}\right)}\right\rangle S\left(p, k_{m}, s-s^{\prime}\right) S\left(p, k_{n}, s-s^{\prime}\right),
\end{aligned}
$$

where the function $S$ (which depends only on even powers of $p$ ) is given in Eq. (2.29). In order to proceed, we approximate $R(s)$ as a Brownian motion as formulated in Eqs. (4.15) and (4.22). We recall that, in the bulk, this approximation holds at all times, whereas it requires $t \ll 1 /(D A \kappa)$ in the case of a film. Next, we replace in Eq. (4.39), as well as in all other contributions stemming from Eq. (4.21), the characteristic function by the expression in Eq. (4.17a) (setting $Q=k_{m}+k_{n}$ ). After performing the time integrals, one obtains in total for a film with periodic BCs

$$
\mathcal{M}_{f}^{(\mathrm{p})}(t)=\frac{K_{d-1}}{L^{2}} \sum_{m, n=-\infty}^{\infty} \int_{0}^{\infty} d p \mathcal{G}^{(\mathrm{p})}\left(k_{n}^{(\mathrm{p})}, k_{m}^{(\mathrm{p})}, p, t\right)
$$

with

$$
\begin{aligned}
\mathcal{G}^{(\mathrm{p})} & =\left[t-\frac{\left(1-e^{\left.-\mathcal{E}^{(\mathrm{p})}\right)}\right.}{\mathcal{E}^{(\mathrm{p})}}\right] \frac{p^{d-2}\left[-2 k_{m} k_{n}\left(2 k_{n}^{2}+p^{2}\right)+k_{m}^{2}\left(3 k_{n}^{2}+2 p^{2}\right)+2 p^{2} k_{n}^{2}+2 k_{n}^{4}+p^{4}\right]}{\mathcal{E}^{(\mathrm{p})}\left(k_{m}^{2}+p^{2}\right)\left(k_{n}^{2}+p^{2}\right)}, \\
\mathcal{E}^{(\mathrm{p})} & =2 D k_{m} k_{n}+k_{m}^{2}\left(2 p^{2}+D\right)+k_{m}^{4}+k_{n}^{2}\left(2 p^{2}+D\right)+k_{n}^{4}+2 p^{4},
\end{aligned}
$$

and where $K_{d}$ is defined in the context of Eq. (3.38). The corresponding expression for $\mathcal{M}_{b}$ is given by replacing in Eq. (4.40) the sums over $k_{m, n}$ by integrals according to

$$
\frac{1}{L} \sum_{j=-\infty}^{\infty} f\left(k_{j}=2 \pi j / L\right) \rightarrow \int_{-\infty}^{\infty} \frac{d k}{2 \pi} f(k)
$$

which holds for any function $f(k)$.

For a film with Neumann BCs, $\mathcal{M}_{f}^{(\mathrm{N})}$ follows from using Eq. (2.32b) in Eq. (3.10) and then by proceeding analogously to the calculation which leads to Eq. (4.40). We note that here both variants of the characteristic function in Eq. (4.17) are needed and that the resulting expression has to be evaluated with $2 L$ instead of $L$. Since the result is rather lengthy it is not reported here. With the exception of the short- and long-time limits (see below), $\mathcal{M}_{f, b}$ have to be determined numerically.

\section{Bulk system}

In order to calculate Eq. (4.40) for a bulk system, in the integrals we rescale all momenta by $t^{-1 / 4}$ and regularize the integral over $p$ by replacing its lower and upper integration limits by $\lambda$ and $\Lambda$, respectively. The resulting expression is independent of $D$ in the limit $t \ll D^{-2}$, which we identify as the short-time regime for the bulk. In this regime one finds the scaling behavior (up to possible logarithmic corrections, see below)

$$
\mathcal{M}_{b}\left(t \ll D^{-2}\right) \simeq t^{\frac{7-d}{4}} \mathcal{W}
$$

with

$$
\mathcal{W}=\int_{\lambda^{*}}^{\Lambda^{*}} d P w(P),
$$

where the (dimensionless) integrand behaves as $w(P \rightarrow 0) \propto$ $P^{d-3}$ and $w(P \rightarrow \infty) \propto P^{d-4}$. The dimensionless integration boundaries $\lambda^{*}$ and $\Lambda^{*}$ in Eq. (4.43) are related to the physical momenta $\lambda$ and $\Lambda$ via $\left\{\lambda^{*}, \Lambda^{*}\right\}=\{\lambda, \Lambda\} t^{1 / 4}$. In $d=3$, the integral in Eq. (4.43) is finite for $\lambda^{*} \rightarrow 0$ but diverges logarithmically upon increasing the value of the upper cutoff $\Lambda^{*} \rightarrow \infty$. By numerically determining the integral in $d=3$ with $\lambda=0$, we find

$$
\mathcal{W} \simeq 0.048+0.011 \times \ln \left(t \Lambda^{4}\right)
$$

Accordingly, in $d=3$, the algebraic time dependence in Eq. (4.42) is modified by a weak logarithmic correction. In $d=2$, instead, a logarithmic correction is induced by the 
infrared divergence of $w$. (For $d=1$ a separate calculation is required.)

\section{Film with periodic $B C s$}

We now consider a film boundary fluctuating near a fixed wall and note that, for $t \ll L^{4}$, the exponential in Eq. (4.40b) varies mildly over a wide range of momenta. Accordingly, one can approximate the sums in $\mathcal{M}_{f}^{(\mathrm{p})}$ in Eq. (4.40) by integrals and, as above, rescale all momenta by $t^{-1 / 4}$. As before, we additionally consider the regime $t \ll 1 /(D A \kappa)$ and note that, consequently, $t \ll D^{-2}$ is automatically fulfilled owing to the adiabatic approximation $D \ll L^{-2}$. The short-time regime for $\mathcal{M}_{f}^{(\mathrm{p})}$ turns out to coincide with Eq. (4.42), i.e.,

$$
\mathcal{M}_{f}^{(\mathrm{p})}\left(t \ll L^{4}\right) \simeq t^{\frac{7-d}{4}} \mathcal{W},
$$

with $\mathcal{W}$ given by Eqs. (4.43) and (4.44).

\section{Film with Neumann BCs}

A scaling analysis of the expression $\mathcal{M}_{f}^{(\mathrm{N})}$ (not reported here) yields, analogously to Eq. (4.45) and under the assumption $t \ll 1 /(D A \kappa)$, the short-time behavior

$$
\mathcal{M}_{f}^{(\mathrm{N})}\left(t \ll L^{4}\right) \simeq t^{\frac{7-d}{4}} \mathcal{W}^{(\mathrm{N})}
$$

with

$$
\mathcal{W}^{(\mathrm{N})}=\int_{\lambda^{*}}^{\Lambda^{*}} d P w^{(\mathrm{N})}(P) \stackrel{d=3}{\simeq} 0.087+0.02 \times \ln \left(t \Lambda^{4}\right) .
$$

As before, the physical momentum cutoffs $\lambda$ and $\Lambda$ enter via $\left\{\lambda^{*}, \Lambda^{*}\right\}=\{\lambda, \Lambda\} t^{1 / 4}$, and the function $w^{(\mathrm{N})}(P)$ behaves asymptotically in the same way as $w$ in Eq. (4.43). As it was the case for a bulk system and for the one with periodic BCs [see Eqs. (4.42) and (4.45)], the algebraic increase of $\mathcal{M}_{f}^{(\mathrm{N})}(t)$ at small times is modified by a weak, logarithmic time dependence.

\section{Integrability of the pressure correlations}

Here, we elucidate the connection between the MSD and the pressure correlations $\langle\Delta \mathcal{P}(t) \Delta \mathcal{P}(0)\rangle$ as determined in Sec. IIIC (see, in particular, Fig. 2). Instead of using Eq. (4.40), the MSD [Eqs. (4.12) and (4.23)] can be obtained in an approximate way by noting that the probability distribution $p\left(R_{s}, R_{s^{\prime}}, R_{0}, s, s^{\prime}\right)$ in Eq. (4.16) is strongly peaked for sufficiently short times $s, s^{\prime}$ and exhibits the maximal statistical weight for $R_{s} \approx R_{s^{\prime}}$. Accordingly, in the short-time regime,

the pressure correlations entering into Eqs. (4.13), (4.14), and (4.21) can be estimated as $\left\langle\Delta \mathcal{P}(R(s), s) \Delta \mathcal{P}\left(R\left(s^{\prime}\right), s^{\prime}\right)\right\rangle \sim$ $\left\langle\Delta \mathcal{P}\left(s-s^{\prime}\right) \Delta \mathcal{P}(0)\right\rangle$ in terms of the pressure correlations at a fixed boundary position as calculated in Sec. III C. Within this approximation, an algebraic behavior $\langle\Delta \mathcal{P}(t) \Delta \mathcal{P}(0)\rangle \propto t^{-\alpha}$ implies $\mathcal{M} \propto t^{2-\alpha}$, provided $\alpha<2$. For $\alpha \geqslant 2$, instead, the pressure correlations are not integrable. Specifically, for the short-time expressions reported in Eqs. (3.34), (3.40), and (3.47d) one has $\alpha=(d+1) / 4$ and thus recovers the algebraic behaviors in Eqs. (4.42), (4.45), and (4.46).

\section{E. Long-time behavior of the MSD}

We now turn to a discussion of the MSD of a movable boundary and of the influence of the CCF on it at long times.

\section{Bulk system}

The long-time behavior of a boundary in a bulk system is provided by Eq. (4.12) together with Eq. (4.40) [after making the appropriate replacement indicated in Eq. (4.41)]. According to the analysis leading to Eq. (4.42), a long-time regime emerges for times $t \gg D^{-2}$. In this limit, the exponential term in Eq. (4.40b) turns out to be negligible, and the remaining expression renders a linear time dependence:

$$
\mathcal{M}_{b}\left(t \gg D^{-2}\right) \simeq t \int_{\lambda}^{\Lambda} d p v(p) .
$$

In order to assess the relevance of the cutoffs $\lambda$ and $\Lambda$, we note that the integrand behaves as $v(p \rightarrow \infty) \propto p^{d-4}$, while the behavior at small $p$ depends in general on $D$. For $D \rightarrow 0$, one has $v \propto p^{d-4}$, while at nonzero $D$, a numerical analysis indicates a behavior close to $v \propto p^{d-3}$. In spatial dimension $d=3$ with nonzero $D$, one may thus set $\lambda=0$, while retaining a logarithmic dependence on the microscopic length scale $\varepsilon \propto 1 / \Lambda$ via the dimensionless combination $D \varepsilon^{2}$. Since the function $v$ depends on the diffusivity $D$, in general the calculation of Eq. (4.48) requires a numerical approach (see $\mathrm{Sec}$. IV F for further discussions).

\section{Film with periodic $B C s$}

In order to determine the long-time behavior of the MSD of a fluctuating boundary in proximity to another wall, we invoke the Markovian approximation discussed in Sec. IV C 2, which leads to an Ornstein-Uhlenbeck process for the boundary position [see Eqs. (4.28), (4.32), and (4.33)]. Equations (4.32), (4.33), and (4.35) imply at long times a static Gaussian distribution with variance [30]

$$
\left\langle[\Delta R]^{2}\right\rangle_{\infty} \equiv\left\langle[\Delta R(t \rightarrow \infty)]^{2}\right\rangle= \begin{cases}\frac{1}{\kappa A}+\frac{D A \mathcal{N}}{2 \kappa}, & \text { (passive) } \\ \frac{1}{\kappa A}, & \text { (thermalized) }\end{cases}
$$

which depends on whether the boundary is passive or thermalized; $\mathcal{N}$ is given by Eqs. (4.31a) and (4.30). The expression in Eq. (4.49b) follows equivalently from Eq. (4.34) because, in fact, the FPE in Eq. (4.33) has been constructed as to yield this result. This term is determined by the variance of the thermal white noise $\eta$ and the curvature $\kappa$ of the effective potential [see Eq. (4.7)], which includes the competing contributions of the electrostatic repulsion and of the $\mathrm{CCF}$ (the factor $1 / A$ arises because the associated force $-\mathcal{U}^{\prime}$ is defined per area). We note that the temperature enters this expression only through $\kappa$ via the temperature dependence of the scaling function of the CCF [see Eq. (4.3)]. The second term in Eq. (4.49a) stems from the fluctuations of the fluctuation-induced force. For a passive boundary they are not balanced by a corresponding 


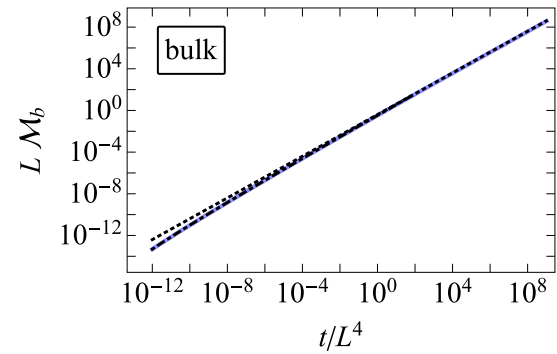

(a)

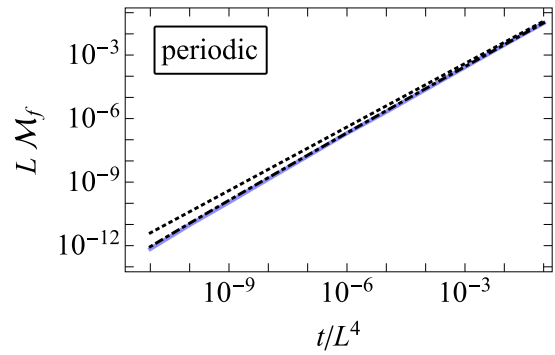

(b)

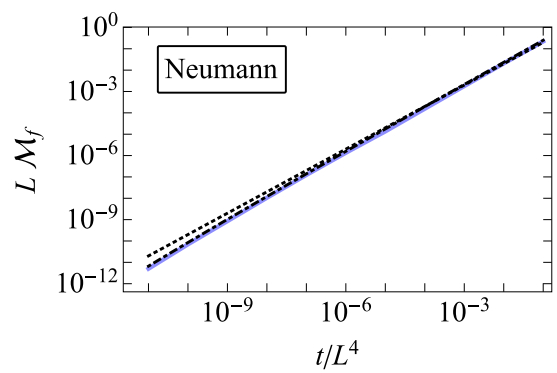

(c)

FIG. 3. Contributions $\mathcal{M}_{b, f}(t)$ [Eqs. (4.14) and (4.21)] to the mean-squared displacement of the movable film boundary at position $R(t)$ [see Eqs. (4.23) and (4.12)] in $d=3$ for (a) a bulklike system $[R(t) \gg L]$, (b) a film with periodic BCs, and (c) a film with Neumann BCs. The solid curves are the results of the numerical calculation of Eq. (4.40) (with analogous expressions for the bulk and the film with Neumann BCs, see the main text), while the dashed-dotted curves (which practically overlap with the solid curves) represent the short-time scaling prediction reported in Eqs. (4.42), (4.45), and (4.46). The dotted lines represent the function $0.37 \times t$, obtained from a numerical evaluation of Eq. (4.48), which describes the long-time behavior of $\mathcal{M}_{b}$. The slight deviations from the behavior $\propto t$ at short times are due to the logarithmic correction in Eqs. (4.44) and (4.47). For illustrative purposes, we have used the value $\Lambda \approx 1000 / \mathcal{L}$ for the large momentum cutoff and $D=0.1 / \mathcal{L}^{2}$ for the bare diffusion constant. The curves depend only very weakly on the choices for these values.

friction term and thus contribute to the steady-state variance. In contrast, kinetic coefficients such as $D$ in general do not enter into steady-state equilibrium quantities.

Specifically for $d=3$ and at bulk criticality $(\tau=0)$, Eq. (4.49a) leads to

$$
\begin{aligned}
\left\langle[\Delta R]^{2}\right\rangle_{\infty}^{\text {pass }} \simeq & \frac{1}{\kappa A}+\frac{D}{\kappa}[0.46-0.042 \times \ln (\lambda / \Lambda) \\
& \left.+0.040 \times(L \lambda)^{-2}\right]
\end{aligned}
$$

where $\lambda$ and $\Lambda$ denote small- and large-momentum cutoffs which stem from the integral over the lateral momentum in Eq. (4.30). Upon naturally identifying the largest length scale $\mathcal{L}$ in the system as $\mathcal{L} \simeq A^{1 / 2}$, such that $\lambda \propto A^{-1 / 2}$, and upon introducing a microscopic (molecular) length $\varepsilon \sim 1 / \Lambda$ (specified below), Eq. (4.50) can be expressed as

$$
\begin{aligned}
\left\langle[\Delta R]^{2}\right\rangle_{\infty}^{\text {pass }} \simeq & \frac{1}{\kappa A}\left\{1+D A\left[0.46+0.042 \times \ln \left(A^{1 / 2} / \varepsilon\right)\right.\right. \\
& \left.\left.+0.040 \times \frac{A}{L^{2}}\right]\right\} .
\end{aligned}
$$

We remark that, off criticality, the term proportional to $A / L^{2}$ in the square brackets disappears owing to the much weaker divergences of the expressions in Eqs. (4.36) and (4.37) for $\tau>0$.

Equation (4.49b) provides a first approximation for the variance of the position of a colloidal particle close to a wall and immersed in a nearly critical solvent in thermal equilibrium, consistent with previous studies $[5,8,28]$. Experimentally, the typical magnitude of the fluctuations is of the order of $\left\langle[\Delta R]^{2}\right\rangle_{\infty}^{1 / 2} \approx 10 \mathrm{~nm}[5]{ }^{14}$ In order to evaluate Eq. (4.51), which is experimentally less relevant due to the underlying passive boundary approximation, we take $A$ to be the cross-sectional area of a typical colloidal particle, $A \simeq(1 \mu \mathrm{m})^{2}$, and estimate the molecular length scale of the solvent as $\varepsilon \simeq 1 \mathrm{~nm}$, in which case Eq. (4.51) reduces to $\left\langle\Delta R^{2}\right\rangle_{\infty}^{\text {pass }} \simeq \frac{1}{\kappa A}(1+0.75 \times D A)$. Because Eq. (4.8) (with $\mathcal{L} \simeq A^{1 / 2}$ ) implies $D A \ll 1$ in the adiabatic limit, the contribution to $\left\langle[\Delta R]^{2}\right\rangle_{\infty}^{\text {pass }}$ stemming from the fluctuations of the CCF is subdominant relative to those stemming from the thermal white noise in Eq. (4.28).

\section{Film with Neumann BCs}

If the fluctuating film boundary is coupled to the OP via Neumann BCs, one obtains at long times the same expression for the variance $\left\langle\Delta R^{2}\right\rangle_{\infty}$ as in Eq. (4.49), but with $\mathcal{N}$ based on Eq. (4.31b) (with $R=0$ ). Specifically, for $d=3$ and $\tau=0$ we obtain, by using the same estimates as in Eq. (4.51), the long-time variance

$$
\left\langle[\Delta R]^{2}\right\rangle_{\infty}=\left\{\begin{array}{lr}
\frac{1}{\kappa A}\left\{1+D A\left[0.85+0.042 \times \ln \left(A^{1 / 2} / \varepsilon\right)+0.020 \times \frac{A}{L^{2}}\right]\right\} & \text { (passive) } \\
\frac{1}{\kappa A} & \text { (thermalized) }
\end{array}\right.
$$

Accordingly, also for Neumann BCs the fluctuations of the CCF yield a subdominant contribution to $\left\langle[\Delta R]^{2}\right\rangle_{\infty}$ for a passive boundary [Eq. (4.52a)].

\footnotetext{
${ }^{14}$ This implies a value $\kappa \approx 10^{28} \mathrm{~m}^{-4}$ [see Eq. (4.7)].
}

\section{F. Discussion}

Figure 3 illustrates the behavior of the critical-fluctuation induced contributions $\mathcal{M}_{b, f}$ to the MSD [Eqs. (4.12) and (4.23)] of a movable film boundary in the adiabatic limit $\left[\chi=D \mathcal{L}^{2} \ll 1\right.$, see Eq. (4.8)], at bulk criticality $(\tau=0)$ and in $d=3$. Figure 3(a) shows the contribution $\mathcal{M}_{b}$ [Eq. (4.14)] 
due to the bulk pressure fluctuations, while Figs. 3(b) and 3(c) illustrate the contributions $\mathcal{M}_{f}$ [Eq. (4.21)] stemming from the pressure fluctuations of a film with periodic or Neumann BCs, respectively. The solid curve in Fig. 3(b) represents the numerical determination of the full expression in Sec. 4.40 for periodic BCs. The same presentation is given for the bulk and for a film with Neumann BCs. These full results are captured accurately by the asymptotic short-time approximations obtained in Eqs. (4.42), (4.45), and (4.46) (dashed-dotted curves, overlapping with the solid ones). We note that, in the bulk as well as in general at short times, the MSD of a passive and a thermalized boundary behave identically in a first approximation [see the discussion around Eq. (4.33)]. According to Eqs. (4.12) and (4.23), in the short-time limit the MSD in $d=3$ amounts to

$$
\left\langle\left[\Delta R\left(t \ll \mathcal{L}^{4}\right)\right]^{2}\right\rangle \simeq 2 D t\left\{1+\chi A \mathcal{L}^{-2}\left[a+b \ln \left(t \Lambda^{4}\right)\right]\right\},
$$

where the constants $a$ and $b$ follow from Eqs. (4.44) and $(4.47)$ as $a_{b}=a^{(\mathrm{p})} \simeq 0.048, a^{(\mathrm{N})} \simeq 0.068, b_{b}=b^{(\mathrm{p})} \simeq 0.011$, $b^{(\mathrm{N})} \simeq 0.016$ for the respective BCs as well as for the bulk (indicated by the superscripts and subscripts). Equation (4.53) applies under the additional assumption $t \ll 1 /(D A \kappa)$, which is equivalent to $D t \ll\left\langle[\Delta R]^{2}\right\rangle_{\infty}$ [obtained by using Eq. (4.49) in the adiabatic limit], as expected for the short-time regime. Taking the largest length scale in the system to be $\mathcal{L} \sim A^{1 / 2}$ (in $d=3$ ), the correction $\propto \chi$ in Eq. (4.53) stemming from the fluctuations of the CCF is weak compared to the Brownian diffusion $2 D t$ induced by the thermal white noise.

At short times, the MSD behaves identically (up to constant prefactors) in the bulk and in proximity to another wall. This is physically expected because the influence of the wall requires a certain amount of time to reach the moving boundary. The data in Fig. 3(a) also cover the long-time regime of the bulk contribution $\mathcal{M}_{b}$, which, according to Eq. (4.48), grows linearly upon increasing $t$ and depends logarithmically on a microscopic length scale. While Eq. (4.53) applies to $d=3$, for $d>3$, the contribution $\propto \chi$ to the MSD scales as $\mathcal{M}_{b, f} \propto t^{(7-d) / 4}$ without a logarithmic correction [see Eqs. (4.42), (4.45), (4.46)] and is dominated by the Brownian contribution $2 D t$ in Eq. (4.53). For $d \leqslant 2$, the correction terms $\mathcal{M}_{b, f}$ depend stronger than linearly on time and additionally acquire logarithmic $(d=2)$ or algebraic dependencies $(d=1)$ on the system size, indicating a potentially anomalous diffusion regime. The corresponding analysis is left for future studies.

At long times $t \gg 1 /(D A \kappa)$, a movable boundary close to a wall explores the effective potential. Within the harmonic approximation, the position variance attains the value given by Eq. (4.49) and (4.52). Within the Markovian approximation, in fact an analytical expression for the full time dependence of the variance of the position follows from Eq. (4.32):

$$
\left\langle[\Delta R(t)]^{2}\right\rangle=\left\langle[\Delta R]^{2}\right\rangle_{\infty}[1-\exp (-2 D A \kappa t)],
$$

with $\left\langle[\Delta R]^{2}\right\rangle_{\infty}$ given by Eq. (4.49) [together with Eq. (4.30), evaluated for $R=0]$. At short times $t \ll 1 /(D A \kappa)$, Eq. (4.54) reduces to $\left\langle[\Delta R(t \ll 1 /[D A \kappa])]^{2}\right\rangle \simeq 2 D t\left(1+D A^{2} \mathcal{N}\right)$, which describes a standard Brownian motion with an effective diffusivity $D+(D A)^{2} \mathcal{N}$. In contrast, the non-Markovian character of the noise $\Delta \mathcal{K}$ in general induces an algebraic short-time be- havior [with possible logarithmic corrections, see Eqs. (4.42), (4.45), (4.46), and (4.53)]. The MSD depends only weakly on these differences because in both cases it is dominated, within the adiabatic approximation, by the Brownian diffusivity $D$.

\section{SUMMARY, CONCLUSIONS, AND OUTLOOK}

In this study, we have analyzed the fluctuations of the critical Casimir force (CCF) acting on the boundary of a film with periodic, Neumann, or Dirichlet BCs (Fig. 1). Our predictions are obtained within a time-dependent GinzburgLandau description of an equilibrium fluid system with a conserved order parameter (model B [35]) and based on a rigorous definition of the instantaneous CCF [9,32-34]. We have considered a film consisting of a spatially fixed boundary and a parallel boundary which is either fixed (see Secs. III B and III C) or movable (see Sec. IV). The movable boundary is coupled "passively" to the OP (see Sec. IV A) and is subject to the action of thermal white noise and the fluctuating CCF. Due to the nature of the passive coupling, the boundary acts on the OP only by imposing BCs, but not by exerting additional friction. By utilizing the fluctuation-dissipation relation, we have shown how this approximation can be improved in order to describe a boundary in thermal equilibrium (see Sec. IV C 2). We have distinguished between the motion in a bulklike environment and the motion close to that boundary of the film which is kept fixed. In this latter case, an effective confinement potential arises due to the attractive mean part of the CCF and a short-ranged electrostatic repulsion. This situation is analogous to a CCF-induced trapping of a colloid close to a solid wall, i.e., the inner boundary $[5,27,28]$.

Our results can be summarized as follows:

(1) The equilibrium variance of the CCF acting on a fixed boundary [Eq. (3.19)] strongly depends on a microscopic cutoff length $\varepsilon$ and diverges in the continuum limit $\varepsilon \rightarrow 0$ [see Eq. (3.31)], consistent with previous studies $[16,17]$. The cutoff $\varepsilon$ is determined by the length scale below which the continuum description of the fluid breaks down. The cutoffdependent terms stem from both the film and the bulk pressure contribution.

(2) The dynamic correlation function of the $\mathrm{CCF}$ [Eq. (3.33)] is finite at nonzero time differences and decays algebraically upon increasing time (see Fig. 2). The short- and long-time scaling behaviors have been calculated analytically for general dimensionality $d$ and general values of the reduced temperature (see Secs. III C 2 and III C 3). In a film in spatial dimension $d=3$, a weak logarithmic divergence is present at short times [see Eqs. (3.43) and (3.47e)], which is cut off by the system size.

(3) The motion of the film boundary is described by a Langevin equation [Eq. (4.2)] which takes into account the random forces emerging from both the fluctuating Casimir force and the thermal fluctuations due to the presence of the heat bath provided by the solvent. The Langevin equation can be solved perturbatively in the adiabatic limit, in which the order-parameter field relaxes much faster than the fluctuating boundary. This solution is characterized by the following features:

(1) In spatial dimension $d=3$ and at short times, the mean-squared displacement (MSD) $\left\langle\Delta R^{2}(t)\right\rangle$ of the 
movable boundary increases linearly in time with a weak logarithmic correction [see Eq. (4.53) and Fig. 3]. The contribution to the MSD stemming from the fluctuations of the CCF is typically small compared to the Brownian diffusion induced by the character of the fluid as a thermal bath.

(2) In the case of a movable boundary which diffuses far from a wall, the Langevin equation yields a Brownian diffusive growth at long times, with a MSD $\propto t$ [see Fig. 3(a)].

(3) In order to assess the long-time behavior of a boundary fluctuating close to a wall, we have derived a Fokker-Planck equation for the probability distribution of the boundary position, based on a Markovian noise approximation [see Eq. (4.32)]. At long times, this equation predicts a steady Gaussian distribution of the boundary position with the variance given by Eq. (4.49). Within the passive boundary approximation, the variance acquires contributions from the fluctuations of the CCF [see Eq. (4.49a)]. By contrast, these contributions are absent in the case of a boundary in thermal equilibrium [see Eq. (4.49b)]; the variance of its position is solely determined by the critical Casimir potential $[5,23,28]$.

The formally divergent equilibrium variance of the $\mathrm{CCF}$ [see Eq. (3.31)] can be considered as an artifact of the corresponding continuum field theory, which cannot be observed directly. In fact, in previous studies of (critical) Casimir forces $[16,29]$, it has been shown that this divergence problem is mitigated or even absent if time-averaged quantities are considered. In this study, we have instead directly linked the statistical properties of the CCF to experimentally observable quantities, such as the position of a film boundary. Our analysis renders a position variance which is finite and reduces to the variance of a Brownian particle in an effective potential set by the mean CCF. This finding is consistent with previous experiments on CCFs in wetting films [19-22] or in colloidal systems [5,23]. Heuristically, this situation is analogous to the fact that observable quantities obtained from a standard Langevin equation are usually finite, despite the random force being correlated $\propto \delta\left(t-t^{\prime}\right)$, which formally diverges in the static limit $t \rightarrow t^{\prime}$. In the present case, the force correlations diverge algebraically (see Fig. 2) but are still integrable in the sense of the MSD (see the discussion in Sec. IV D 5). Moreover, due to the limited temporal resolution of a measurement device, experimental measurements are typically not sensitive to fluctuations of the CCF at frequency scales pertaining to the relaxation of the short-wavelength modes [16].

Our analysis of the dynamics of the boundary is based on two central assumptions: first, the moving boundary acts on the OP only via the imposed BCs, not via coupling terms in the equation of motion (passive boundary approximation) $[24,25,47]$. While, within this approximation, detailed balance is broken and the system is inherently out of equilibrium, we have also shown how to reestablish detailed balance at the level of the Fokker-Planck equation for the position of the boundary [see Eq. (4.33)]. Second, the relaxation dynamics of the boundary is much slower than the one of the order parameter; this is fulfilled in typical experiments [28] and also ensured in our study because we have assumed a finite, albeit macroscopically large, system size. Within the passive boundary approximation, the actual Casimir potential, which is related only to the mean CCF, cannot be simply inferred by assuming a standard Boltzmann distribution of the position [23] because OP fluctuations render additional contributions to the variance which can vary in space [see Eq. (4.30)]. Asymptotically, in the adiabatic limit these contributions are negligible.

Concerning future research, a systematic derivation of the dynamical equation from a more fundamental model, beyond the passive boundary approximation, would be desirable. It would also be interesting to extend the present analysis towards the dynamics of a spherical colloidal particle which has a finite radius, is immersed in a critical solvent, and floats near a wall [28,52]. It is justified to expect a wealth of additional effects due to hydrodynamic interactions, the nonplanar geometry, and the strong preferential adsorption of the OP at the surfaces $[7,53,54]$.

\section{ACKNOWLEDGMENTS}

A.G. acknowledges financial support from MIUR PRIN project "Coarse-grained description for nonequilibrium systems and transport phenomena (CO-NEST)," Project No. 201798CZL.

\section{APPENDIX: EQUILIBRIUM DISTRIBUTION OF A MOVABLE BOUNDARY}

Here, we discuss the probability distribution of the position of a movable boundary in thermal equilibrium with a critical fluid in a half-space [32,34]. The Hamiltonian of this system is given by $\mathcal{F}(R ;[\phi])=\int_{V} d^{d} r \mathcal{H}(\mathbf{r}, R, \phi(\mathbf{r}), \nabla \phi(\mathbf{r}), \tau)$ [see Eq. (2.2)], where the position $R$ of the boundary is regarded as an additional degree of freedom, which emerges via the surface contribution $\mathcal{H}_{s}$ [Eq. (2.7)]. Accordingly, the joint probability distribution is given by [see Eq. (2.1)]

$$
P_{\mathrm{eq}}([\phi], R) \sim e^{-\mathcal{F}(R ;[\phi]) / T} .
$$

The equilibrium probability distribution of $R$ follows by integrating over $\phi$ :

$$
\begin{aligned}
P_{\mathrm{eq}}(R) & =\frac{\mathcal{Z}(R)}{\mathcal{Z}_{0}}, \quad \mathcal{Z}(R)=\int \mathcal{D} \phi e^{-\mathcal{F}(R ;[\phi]) / T}, \\
\mathcal{Z}_{0} & =\int_{0}^{\infty} d R \mathcal{Z}(R) .
\end{aligned}
$$

The mean equilibrium $\mathrm{CCF}\langle\mathcal{K}\rangle$ (per area $A$ and temperature $T$ ) acting on the boundary [which in Eq. (2.21) is expressed in terms of the averaged stress tensor] can be equivalently obtained from $\mathcal{Z}(R)$ [32]:

$$
A\langle\mathcal{K}(R)\rangle_{\phi}=\frac{d}{d R} \ln \mathcal{Z}(R) .
$$

Upon introducing the critical Casimir potential $V_{C}(R)$ via $\langle\mathcal{K}(R)\rangle_{\phi}=-d V_{\mathrm{C}}(R) / d R$, and by using Eq. (A3), the distribution $P_{\text {eq }}$ in Eq. (A2) can be expressed as

$$
P_{\mathrm{eq}}(R)=\frac{1}{\mathcal{Z}} e^{-A V_{C}(R)} .
$$


Accordingly, if an inclusion is represented in terms of a Hamiltonian interaction with the fluid (as, e.g., in
Refs. $[24,25,47])$, the fluctuations of its position $R$ are actually controlled by the potential of mean Casimir force.
[1] M. Le Bellac, Quantum and Statistical Field Theory (Clarendon, Oxford, 1991).

[2] M. E. Fisher and P. G. de Gennes, Wall phenomena in a critical binary mixture, C. R. Acad. Sci. Paris B 287, 207 (1978).

[3] M. Krech, The Casimir Effect in Critical systems (World Scientific, Singapore, 1994).

[4] J. G. Brankov, D. M. Dantchev, and N. S. Tonchev, The Theory of Critical Phenomena in Finite-Size Systems (World Scientific, Singapore, 2000).

[5] C. Hertlein, L. Helden, A. Gambassi, S. Dietrich, and C. Bechinger, Direct measurement of critical Casimir forces, Nature (London) 451, 172 (2008).

[6] A. Gambassi, The Casimir effect: From quantum to critical fluctuations, J. Phys.: Conf. Ser. 161, 012037 (2009).

[7] A. Furukawa, A. Gambassi, S. Dietrich, and H. Tanaka, Nonequilibrium Critical Casimir Effect in Binary Fluids, Phys. Rev. Lett. 111, 055701 (2013).

[8] A. Maciolek and S. Dietrich, Collective behavior of colloids due to critical Casimir interactions, Rev. Mod. Phys. 90, 045001 (2018).

[9] M. Gross, C. M. Rohwer, and S. Dietrich, Dynamics of the critical Casimir force for a conserved order parameter after a critical quench, Phys. Rev. E 100, 012114 (2019).

[10] C. M. Rohwer, A. Maciolek, S. Dietrich, and M. Krüger, Correlations and forces in sheared fluids with or without quenching, New. J. Phys. 21, 073029 (2019).

[11] A. Callegari, A. Magazzù, A. Gambassi, and G. Volpe, Optical trapping and critical Casimir forces, Eur. Phys. J. Plus 136, 213 (2021).

[12] H. W. Diehl, Field-theoretical Approach to Critical Behavior at Surfaces, in Phase Transitions and Critical Phenomena, Vol. 10, edited by C. Domb and J. L. Lebowitz (Academic, London, 1986), p. 76.

[13] D. Dantchev, M. Krech, and S. Dietrich, Universality of the thermodynamic Casimir effect, Phys. Rev. E 67, 066120 (2003).

[14] D. Dantchev, F. Schlesener, and S. Dietrich, Interplay of critical Casimir and dispersion forces, Phys. Rev. E 76, 011121 (2007).

[15] G. Valchev and D. Dantchev, Sign change in the net force in sphere-plate and sphere-sphere systems immersed in nonpolar critical fluid due to the interplay between the critical Casimir and dispersion van der Waals forces, Phys. Rev. E 96, 022107 (2017).

[16] D. Bartolo, A. Ajdari, J.-B. Fournier, and R. Golestanian, Fluctuations Of Fluctuation-Induced Casimir-Like Forces, Phys. Rev. Lett. 89, 230601 (2002).

[17] D. Dantchev and M. Krech, Critical Casimir force and its fluctuations in lattice spin models: Exact and Monte Carlo results, Phys. Rev. E 69, 046119 (2004).

[18] A.-F. Bitbol, P. G. Dommersnes, and J.-B. Fournier, Fluctuations of the Casimir-like force between two membrane inclusions, Phys. Rev. E 81, 050903(R) (2010).

[19] R. Garcia and M. H. W. Chan, Critical Fluctuation-Induced Thinning Of ${ }^{4} \mathrm{He}$ Films Near The Superfluid Transition, Phys. Rev. Lett. 83, 1187 (1999).
[20] A. Ganshin, S. Scheidemantel, R. Garcia, and M. H. W. Chan, Critical Casimir Force In ${ }^{4} \mathrm{He}$ Films: Confirmation Of FiniteSize Scaling, Phys. Rev. Lett. 97, 075301 (2006).

[21] M. Fukuto, Y. F. Yano, and P. S. Pershan, Critical Casimir Effect In Three-Dimensional Ising Systems: Measurements On Binary Wetting Films, Phys. Rev. Lett. 94, 135702 (2005).

[22] S. Rafaï, D. Bonn, and J. Meunier, Repulsive and attractive critical Casimir forces, Phys. A (Amsterdam) 386, 31 (2007).

[23] A. Gambassi, A. Maciolek, C. Hertlein, U. Nellen, L. Helden, C. Bechinger, and S. Dietrich, Critical Casimir effect in classical binary liquid mixtures, Phys. Rev. E 80, 061143 (2009).

[24] V. Demery and D. S. Dean, Perturbative path-integral study of active- and passive-tracer diffusion in fluctuating fields, Phys. Rev. E 84, 011148 (2011).

[25] D. S. Dean and V. Demery, Diffusion of active tracers in fluctuating fields, J. Phys.: Condens. Matter 23, 234114 (2011).

[26] V. Demery, Diffusion of a particle quadratically coupled to a thermally fluctuating field, Phys. Rev. E 87, 052105 (2013).

[27] S. Paladugu, A. Callegari, Y. Tuna, L. Barth, S. Dietrich, A. Gambassi, and G. Volpe, Nonadditivity of critical Casimir forces, Nat. Commun. 7, 11403 (2016).

[28] A. Magazzù, A. Callegari, J. P. Staforelli, A. Gambassi, S. Dietrich, and G. Volpe, Controlling the dynamics of colloidal particles by critical Casimir forces, Soft Matter 15, 2152 (2019).

[29] G. Barton, On the fluctuations of the Casimir forces. II. The stress-correlation function, J. Phys. A: Math. Gen. 24, 5533 (1991).

[30] C. Gardiner, Stochastic Methods: A Handbook for the Natural and Social Sciences, 4th ed. (Springer, Berlin, 2009).

[31] D. S. Dean, V. A. Parsegian, and R. Podgornik, Fluctuation of thermal van der Waals forces due to dipole fluctuations, Phys. Rev. A 87, 032111 (2013).

[32] D. S. Dean and A. Gopinathan, Out-of-equilibrium behavior of Casimir-type fluctuation-induced forces for free classical fields, Phys. Rev. E 81, 041126 (2010).

[33] M. Krüger, A. Solon, V. Demery, C. M. Rohwer, and D. S. Dean, Stresses in non-equilibrium fluids: Exact formulation and coarse-grained theory, J. Chem. Phys. 148, 084503 (2018).

[34] M. Gross, A. Gambassi, and S. Dietrich, Surface-induced nonequilibrium dynamics and critical Casimir forces for model B in film geometry, Phys. Rev. E 98, 032103 (2018).

[35] P. C. Hohenberg and B. I. Halperin, Theory of dynamic critical phenomena, Rev. Mod. Phys. 49, 435 (1977).

[36] H. W. Diehl and H. K. Janssen, Boundary conditions for the field theory of dynamic critical behavior in semi-infinite systems with conserved order parameter, Phys. Rev. A 45, 7145 (1992).

[37] A. Gambassi and S. Dietrich, Critical dynamics in thin films, J. Stat. Phys. 123, 929 (2006).

[38] A. Gambassi, Relaxation phenomena at criticality, Eur. Phys. J. B 64, 379 (2008).

[39] F. W. J. Olver, D. W. Lozier, R. F. Boisvert, and C. W. Clark, NIST Handbook of Mathematical Functions, 1st ed. (Cambridge University Press, Cambridge, 2010). 
[40] M. Krech and S. Dietrich, Free energy and specific heat of critical films and surfaces, Phys. Rev. A 46, 1886 (1992).

[41] V. Demery and D. S. Dean, Drag forces on inclusions in classical fields with dissipative dynamics, Eur. Phys. J. E 32, 377 (2010).

[42] V. Démery and D. S. Dean, Thermal Casimir drag in fluctuating classical fields, Phys. Rev. E 84, 010103(R) (2011).

[43] R. Mannella and P. V. E. McClintock, Itô versus Stratonovich: 30 years later, Fluct. Noise Lett. 11, 1240010 (2012).

[44] G. A. Pavliotis, Stochastic Processes and Applications: Diffusion Processes, the Fokker-Planck and Langevin Equations, Texts in Applied Mathematics (Springer, New York, 2014).

[45] J. M. Sancho, Brownian colloidal particles: Ito, Stratonovich or a different stochastic interpretation, Phys. Rev. E 84, 062102 (2011).

[46] G. Volpe and J. Wehr, Effective drifts in dynamical systems with multiplicative noise: a review of recent progress, Rep. Prog. Phys. 79, 053901 (2016).

[47] M. Gross, Dynamics and steady states of a tracer particle in a confined critical fluid, arXiv:2101.02072.
[48] M. Gross, A. Gambassi, and S. Dietrich, Statistical field theory with constraints: Application to critical Casimir forces in the canonical ensemble, Phys. Rev. E 96, 022135 (2017).

[49] J. N. Israelachvili, Intermolecular and Surface Forces (Academic, London, 2011).

[50] R. L. Stratonovich, Topics in the Theory of Random Noise (Gordon and Breach, New York, 1963).

[51] R. Kubo, The fluctuation-dissipation theorem, Rep. Prog. Phys. 29, 255 (1966).

[52] O. A. Vasilyev, S. Dietrich, and S. Kondrat, Nonadditive interactions and phase transitions in strongly confined colloidal systems, Soft Matter 14, 586 (2018).

[53] Y. Fujitani, Fluctuation amplitude of a trapped rigid sphere immersed in a near-critical binary fluid mixture within the regime of the gaussian model, J. Phys. Soc. Jpn. 85, 044401 (2016).

[54] S. Yabunaka and Y. Fujitani, Drag coefficient of a rigid spherical particle in a near-critical binary fluid mixture, beyond the regime of the Gaussian model, J. Fluid Mech. 886, A2 (2020). 